THESIS

\title{
A MODEL OF THE EFFECTS OF AUTOMATIC GENERATION CONTROL SIGNAL CHARACTERISTICS ON ENERGY STORAGE SYSTEM RELIABILITY
}

\author{
Submitted by \\ Timothy M. Campbell \\ Department of Mechanical Engineering \\ In partial fulfillment of the requirements \\ For the Degree of Master of Science \\ Colorado State University \\ Fort Collins, Colorado \\ Spring 2012
}

Master's Committee:

Advisor: Thomas H. Bradley

Daniel Zimmerle

Peter M. Young 
ABSTRACT

\section{A MODEL OF THE EFFECTS OF AUTOMATIC GENERATION CONTROL SIGNAL CHARACTERISTICS ON ENERGY STORAGE SYSTEM RELIABILITY}

No electrochemical batteries constructed to date have the storage capacities necessary for integration into conventional energy markets; aggregation will be required to meet industrystandard metrics for reliability and availability. This aggregation of individual energy storage devices into a distributed energy storage (DES) system will be useful not only to allow standard connection to the grid, but to provide higher-quality fast-response grid services with low-cost technologies. These smaller installations will have lower capital costs than traditional energy storage facilities. Ancillary services, and more specifically frequency regulation services, are understood to be the most technically viable and economically valuable market available to DES. Accordingly, this study is based on the properties of the frequency regulation market.

This study presents a simplified model of a DES resource, its frequency regulation actuation signal, and its mode of market participation. The inputs to the model are scaling parameters of the DES system and of the actuation signal. The outputs from the model are the individual and aggregated reliability of the DES system. An analytical calculation of reliability is performed and analytical results are compared to numerical simulation solutions. Results show that the reliability of the energy storage device can be characterized using a set of nondimensional parameters. These device-level reliability results are then translated into systemlevel reliability through several different models of ancillary services contracting and dispatch. 
Previous studies of DES systems have assumed that the energy storage system has no energy storage limitations and that the actuation signal has no net or instantaneous energy content. This model includes these conditions so as to capture the interaction between the energy content of the Automatic Generation Control (AGC) signal and the device-level and system-level reliability of DES systems. These results are novel in that they can guide the independent system operator/balancing authority in constructing an AGC signal specific to the needs of DES system resources. 


\section{ACKNOWLEDGEMENTS}

The work presented herein would not have been possible without the attention and funding provided by the California Energy Commission and the Recovery Act.

My advisor, Thomas Bradley, Ph. D., has been instrumental in not only this research, but my continued education and curiosity of engineering and energy systems.

My family, especially Sarah, Kit, Jerry, Tim, Claire, and Daniel have been supportive of me beyond all reasonable measures, especially in the work leading to the completion of this thesis.

All my friends and colleagues have supported me in one way or another and daily push my intellectual boundaries.

Thank you. 


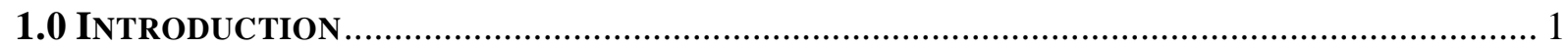

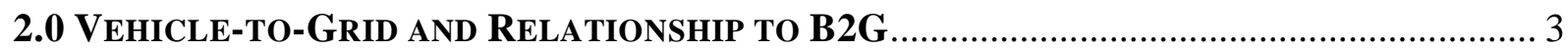

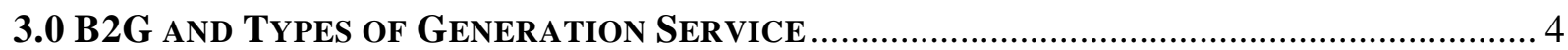

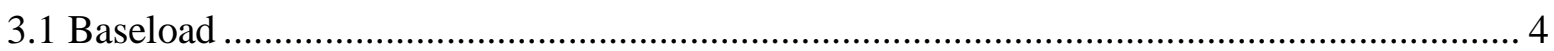

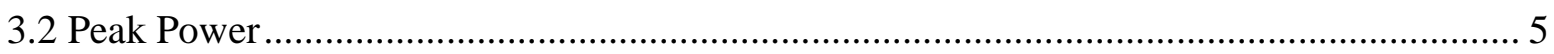

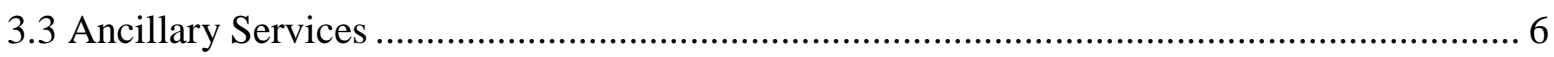

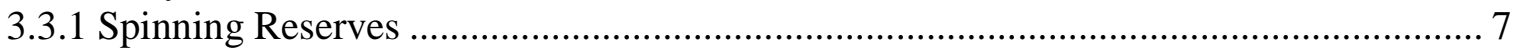

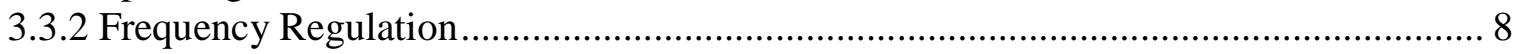

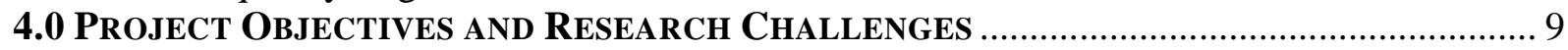

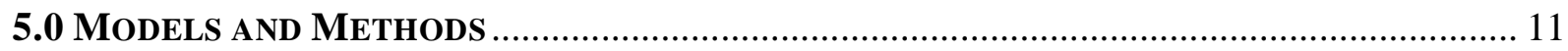

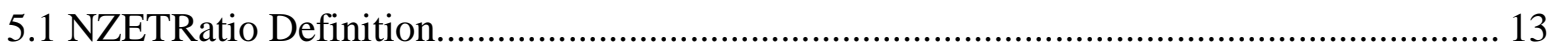

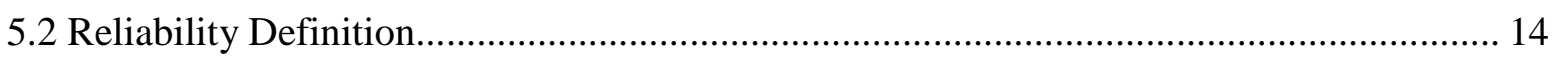

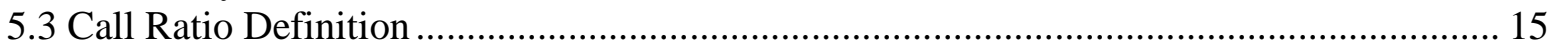

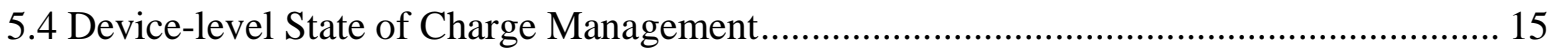

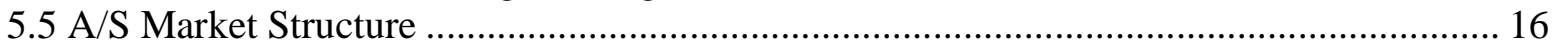

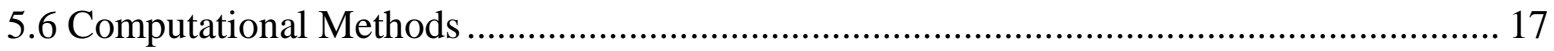

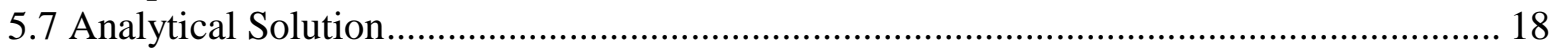

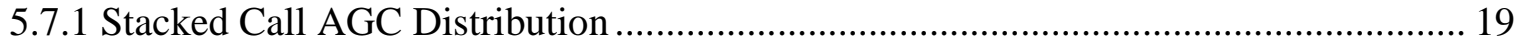

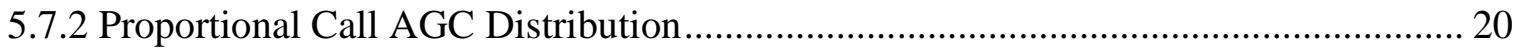

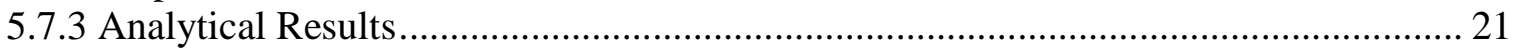

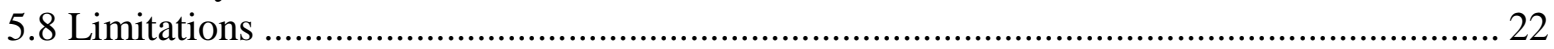

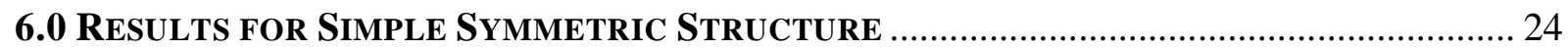

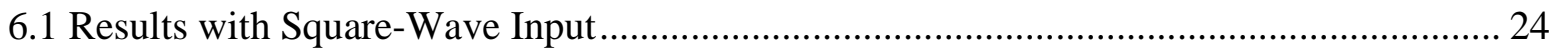

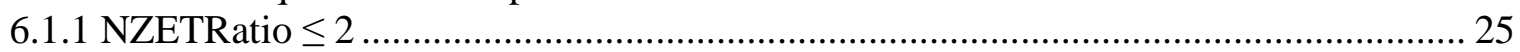

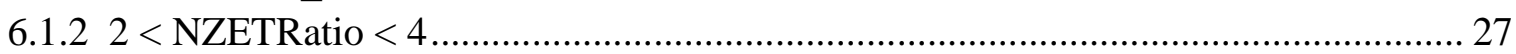

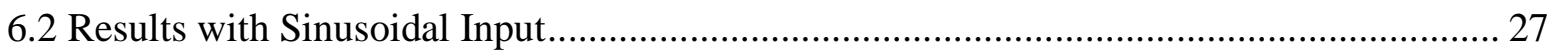

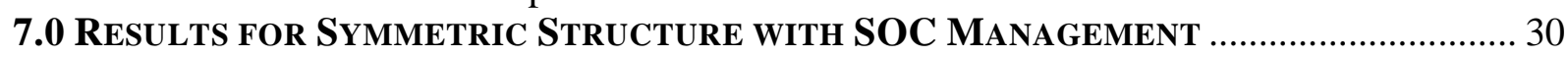

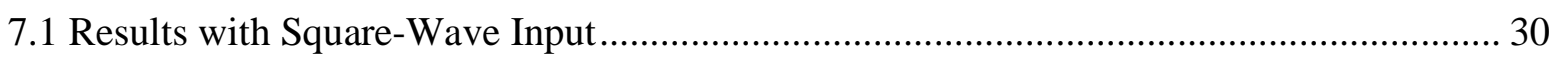

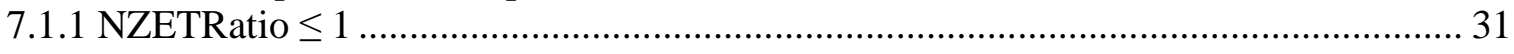

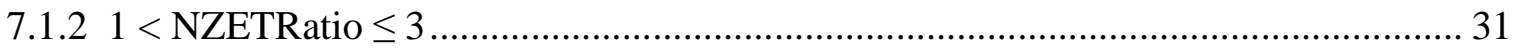

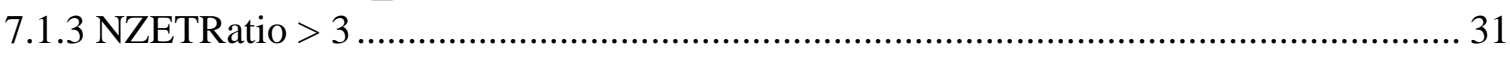

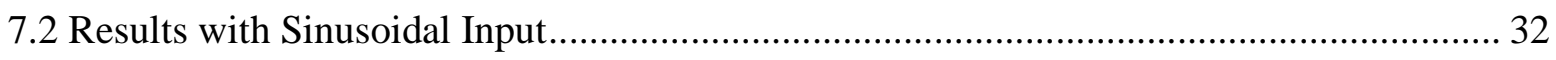

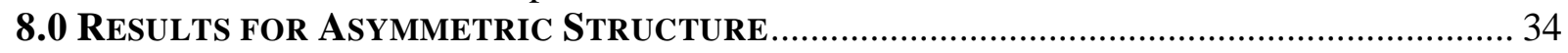

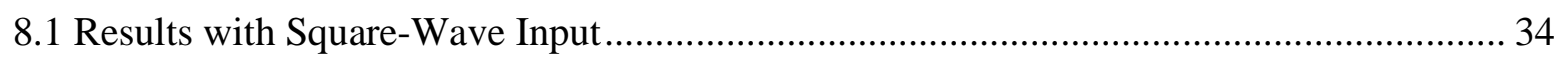

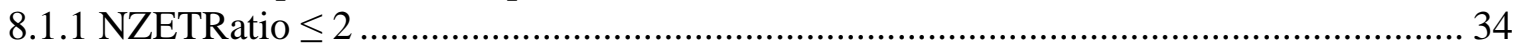

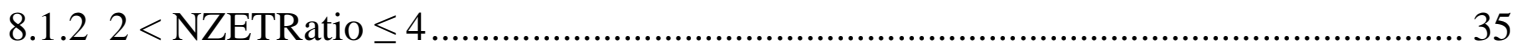

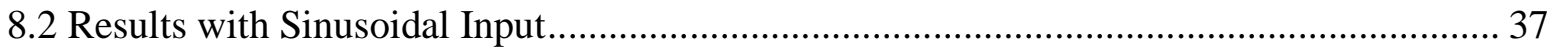

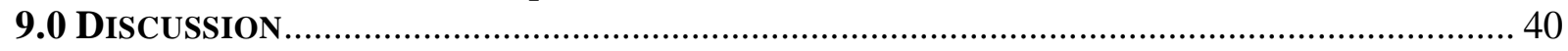

9.1 Evaluation of Methods for Improving ES Device Reliability ....................................... 40

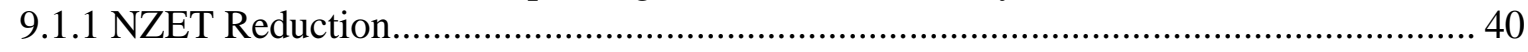

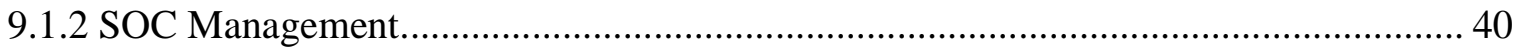

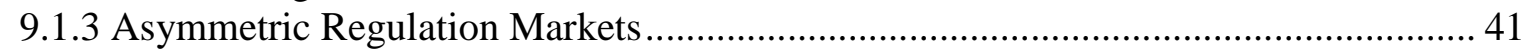

9.2 Synthesizing DES System Reliability with Frequency Regulation ................................... 42 
9.3 Benchmarking Against Currently-Available AGC Signals ............................................. 44

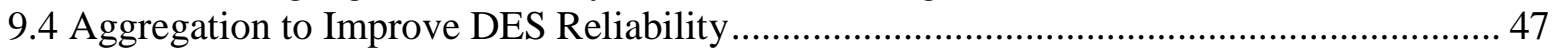

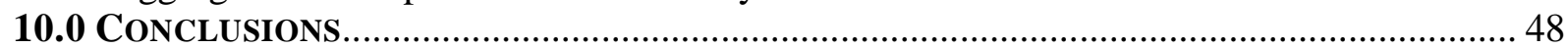

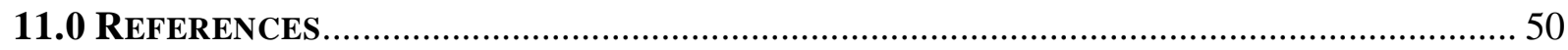

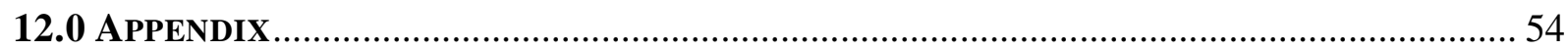

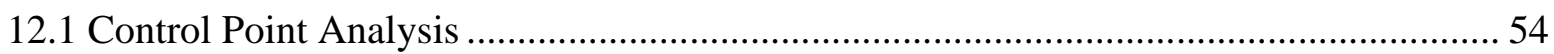

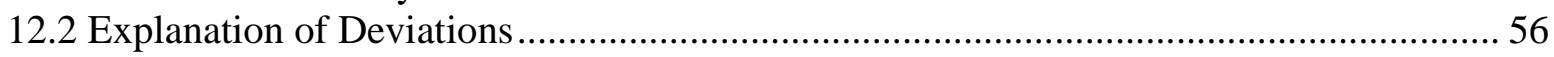




\section{TABLE OF FIGURES}

Fig. 1: Difference in scale between regulation services and generation (as in [6]) ...................... 5

Fig. 2: Stacked call method for DES signal distribution ........................................................ 12

Fig. 3: Proportional call method for DES signal distribution ................................................... 12

Fig. 4: SOC of ES device over time with NZETRatio $=1$ and $100 \%$ call ratio, with the grid

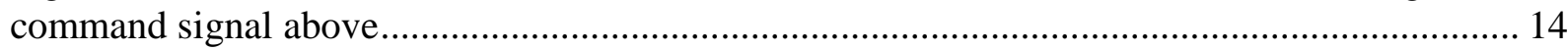

Fig. 5: Position of values a, b, and c for the first net-zero-energy period of a power command

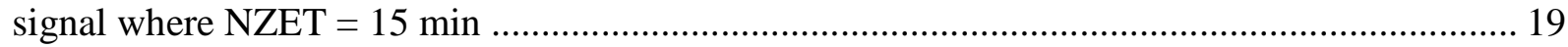

Fig. 6: Analytical solution to reliability of DES at $100 \%$ call ratio........................................ 22

Fig. 7: Reliability vs. NZETRatio for three call ratio values and square-wave input and simple

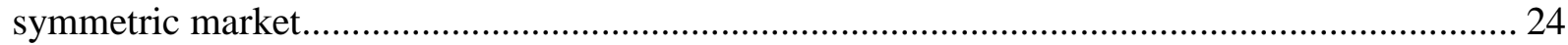

Fig. 8: Reliability vs. NZETRatio for three call ratios and square-wave input with simple

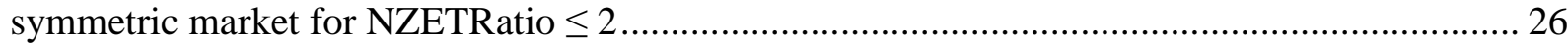

Fig. 9: SOC over time with $100 \%$ call ratio and initial saturation in the disregarded transient period at NZETRatio $=2$

Fig. 10: Reliability vs. NZETRatio for three call ratio values and sinusoidal input with simple symmetric market 28

Fig. 11: Comparison of maximum square-wave reliability with minimum sinusoidal reliability for simple symmetric structure

Fig. 12: Reliability vs. NZETRatio for three call ratio values and square-wave input with SOC management

Fig. 13: Reliability vs. NZETRatio for three call ratio values and sinusoidal input with SOC

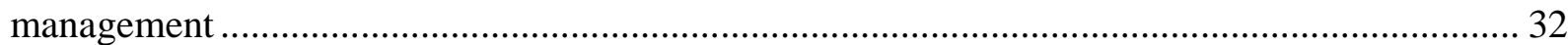
Fig. 14: Reliability vs. NZETRatio for four call ratios and square-wave input with asymmetric market

Fig. 15: Comparison of simple symmetric market and asymmetric market for square-wave input at $10 \%$ call ratio

Fig. 16: Reliability vs. NZETRatio for $80 \%$ call ratio and square-wave input with simple

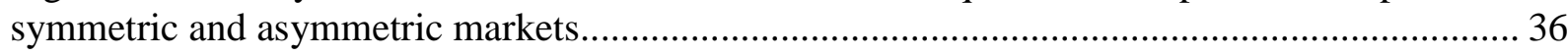

Fig. 17: Reliability vs. NZETRatio for three call ratio values and sinusoidal input with

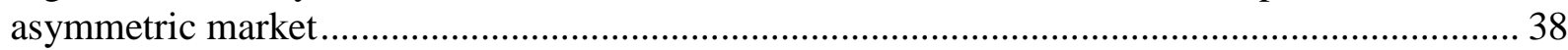

Fig. 18: Comparison of sinusoidal command signal results with asymmetric and simple symmetric markets

Fig. 19: Comparison of symmetric and asymmetric market structures with sinusoidal input and $10 \%$ call ratio

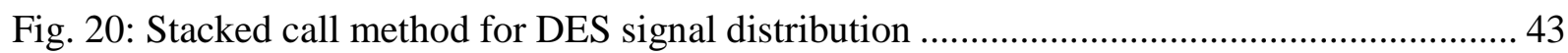

Fig. 21: Proportional call method for DES signal distribution ............................................... 43

Fig. 22: Control point analysis for asymmetric structure study. From top to bottom: $100 \%$ call

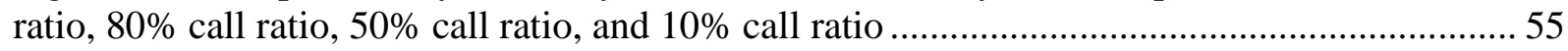

Fig. 23: Analytical explanation of the predictable reliability deviations .................................... 57 


\section{LIST OF KEY TERMS}

ACE - Area Control Error

AGC - Automatic Generation Control

AH - Available Hours

A/S - Ancillary Services

B2G - Battery-to-Grid

BA - Balancing Authority

BEV - Battery-Electric Vehicle

C\&I - Commercial \& Industrial

CAISO - California Independent System Operator

DES - Distributed Energy Storage

EDV - Electric-Drive Vehicle

EFDH - Equivalent Forced Derated Hours

ES - Energy Storage

FO - Frequency Only

HEV - Hybrid-Electric Vehicle

ISO - Independent System Operator

NERC - North American Electric Reliability Corporation

NZET - Net-Zero-Energy Time

NZETRatio - Net-Zero-Energy Time Ratio

PACE - Processed Area Control Error

PHEV - Plug-in Hybrid-Electric Vehicle

$\mathrm{SH}$ - Service Hours

viii 
SOC - State-of-Charge

V2G - Vehicle-to-Grid 


\subsection{INTRODUCTION}

Batteries for automotive use allow hybrid-electric (HEV) or battery-electric (BEV) vehicles to have many advantages over conventional vehicles, such as increased engine efficiency, decreased driving costs, and decreased emissions [1-3]. One of the disadvantages, however, is that a battery can be utilized for transportation only for so long because manufacturers desire driveability and performance to be the same throughout the usable life of the battery [4]. This so-called "end-of-life" for automotive batteries occurs after no more than $20 \%$ energy and power capacity loss for BEVs or $23 \%$ for HEVs [5]. After the batteries have served their useful lives in vehicles, they are currently either discarded or their components recycled. Since there is a significant amount of energy and power capacity left in each battery, a "second-life" usage program has been proposed wherein batteries can be connected to the electric power grid [4]. A second-life program may also offset the initial costs of new batteries [3]. This system is known as Battery-to-Grid.

Battery-to-Grid (B2G) is the idea of using batteries to add energy storage (ES) capacity to the electric power grid. Currently, the grid contains only about $2 \%$ storage; power generation must match power demand at all times [6]. This can be costly at times of high demand because peak generators need to ramp up their output to match demand [7]. ES would permit the grid to store excess energy generated during periods of low demand and to dispatch the energy during high or peak demand periods, allowing traditional generators to operate at a more constant rate [8]. This would increase generator efficiency and reduce operating and maintenance costs to generators by reducing transient operations [9]. Some areas have already integrated ES capacity into their dispatch, typically in the form of pumped hydroelectric generation [7]. Pumped hydroelectric generation is advantageous to the grid because of low costs, near-zero emissions, 
and fast response to peak loads [10]. For areas without access to hydroelectric resources, batteries could serve an ES role.

Another use of ES on the electric grid is for renewables firming. Firming refers to conditioning of the power output of a generator to improve compatibility with the grid [11]. Because renewable resources such as solar and wind have power output that is highly variable, energy storage is necessary to either strictly store energy during low-demand periods when it cannot be used, or to collect energy temporarily until there is enough capacity to be of use in an energy market [7], [12]. B2G is already in use in renewable resource management, such as the current largest lithium-ion B2G system, run by AES Energy Storage, which is connected to a 98MW wind power facility [13]. While renewables firming is an important aspect toward the motivation of energy storage connectivity and specifically B2G, it is not in the scope of this paper and is mentioned only to provide thorough background on potential B2G uses [3], [6], [8], [14], [15].

Currently, ES devices for B2G have been designed and constructed specifically for B2G. By enabling second-life automotive batteries to participate in $\mathrm{B} 2 \mathrm{G}$, the energy storage capacity on the electric grid will rise dramatically [8]. For example, the power capacity of electric-drive vehicles (EDVs) in California in 2008 was estimated to be $4 \%$ of the state's generating capacity [16]. HEVs have already achieved mass commercialization and plug-in hybrid-electric vehicles (PHEVs) are forecast to achieve this by 2015, which will increase the EDV total power capacity [17]. Assuming the connection of a hypothetical future fleet of EDVs at $25 \%$ that of the national fleet, the power capacity of the total electrical system will be doubled, with the energy capacity growing proportionally [12]. In addition, batteries designed for automotive use are especially well suited for energy markets due to their high power and low cost [18]. 


\subsection{VEHICLE-TO-GRID AND RELATIONSHIP TO B2G}

Vehicle-to-Grid (V2G) is the use of energy and power capacity from parked EDVs for stability of the electric grid [12], [19]. V2G and B2G share the concept of bidirectional power flow with the electric grid, as long as vehicles do not provide remote generation through either an internal combustion engine or a fuel cell [7], [19], [20]. Most studies to date have been on the viability of V2G with respect to EDVs such as PHEVs or plug-in fuel cell hybrid-electric vehicles. Many of the cited studies assume remote generation is allowable and generally preferable, but because of potential safety and feasibility concerns, this study considers batterysupplied energy only [21].

$\mathrm{V} 2 \mathrm{G}$ and B2G are also related in that they are distributed resources [20], [22]. For either of these to be viable as grid-attached ES, an aggregator is necessary to combine the relatively small capacities of each vehicle battery into a system large enough to effectively serve the grid [23], [24]. This aggregation allows ES devices to combine their capacities and create a distributed energy storage (DES) system. Once devices have been aggregated into a DES system to meet or exceed the typical $1 \mathrm{MW}$ minimum market requirement, there are a number of energy services that are available for contracting [25]. 


\subsection{B2G AND TyPES OF GENERATION SERVICE}

Many types of generation services in use today have previously been analyzed for viability with V2G. Due to the similarities between V2G and B2G, these analyses are also useful to determine viability of DES systems as B2G resources. The subsequent subsections will outline those services, the potential advantages and disadvantages for B2G and DES systems, and conclusions about each with respect to $\mathrm{B} 2 \mathrm{G}$.

\subsection{BASELOAD}

The baseload service is the most basic generation service in that it provides the majority of energy used at all times (Fig. 1). Because of the constant demand for power, baseload is typically contracted long-term and at a relatively low per-kWh price [18], [22]. Baseload provides near-constant power, which is beneficial for coal-fired, nuclear, hydroelectric, or natural-gas plants as these devices are more efficient at steady-state [7], [18]. Several studies have shown that baseload power is not suitable for production by batteries for a variety of reasons [7], [12], [18], [22], [26]. First of all, the low per-kWh revenue from baseload is much lower than the costs incurred from providing B2G [26]. The durability of batteries is also 10-50 times lower than traditional generators; the constant power requirement would cause accelerated deterioration of $\mathrm{B} 2 \mathrm{G}$ resources [12]. Furthermore, there is opportunity cost associated with baseload power not taking advantage of the quick response time of batteries [22]. These analyses all conclude that baseload power is best generated by traditional means. 


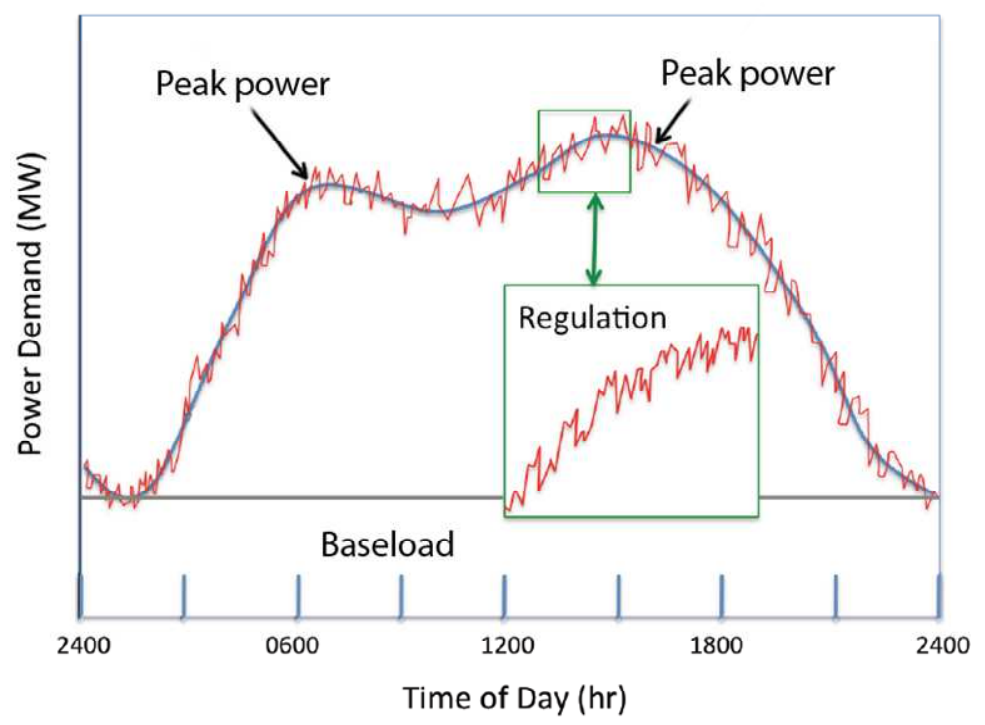

Fig. 1: Difference in scale between regulation services and generation (as in [6])

\subsection{PEAK POWER}

Another generation service considered is that of peak power. Peak power is a type of generation applied only at times when high levels of power consumption are expected (Fig. 1) [22]. Because it is only needed a few hundred hours each year, peak power is traditionally generated by gas generators since they have a shorter startup time and lower capital costs than larger plants [7], [18], [22]. Due to its low total operating time, peak power has been studied for V2G extensively [2], [3], [7], [14], [18], [21], [22]. While earlier studies assumed that peak power would be the primary operation for vehicle batteries, more recent research has had differing results. The early analytical exercise performed by Kempton et al. suggested that peak power would be economically feasible [2]. Kamboj et al. also suggested that vehicle batteries may be suitable for peak power, but their capacities may limit the power that can be provided economically [7]. A later study by Kempton et al. showed that peak power from V2G could be viable if electricity rate schedules are adapted, but at the time of writing, battery vehicles were too costly to run in this service [14]. A still later study by Kempton et al. could not conclude that 
batteries would be viable for peak power because of the price variability of the market [22]. Based on the variation in results, it is reasonable to conclude that peak power is not an immediately viable service in which to participate. Nonetheless, after saturation of more-feasible services, peak power could be revisited [12]. B2G in this area would allow older, less efficient peaking units to retire [21].

A use of B2G similar to peak power is that of peak shaving. Peak shaving is also matching of peak demand to generation, but by demand reduction through generation on the customer side of the meter rather than increases in utility-side generation [27]. General preliminary analyses shows that batteries could be viable for peak shaving [15]. Analysis specific to residential peak shaving shows that certain battery types have low enough costs to allow profit, although never with levelized electric rates. More promising results have been shown in commercial and industrial $(\mathrm{C} \& \mathrm{I})$ applications, where monthly demand charges are based on the peak power used during the month. By lowering the peak power, the largest part of C\&I electricity bills can be decreased. Under conditions of high electricity rates, C\&I peak shaving may be viable. Peak shaving, like peak power, has lower margins than other available services which suggests that it is not the ideal use for $\mathrm{B} 2 \mathrm{G}$, but it has been encouraged in some cases because there are no infrastructure updates necessary [22].

\subsection{ANCILlARY SERVICES}

Ancillary services $(\mathrm{A} / \mathrm{S})$ are energy markets that maintain the integrity and reliability of the electric grid and have lower power and energy requirements than peak power or baseload services [15], [19]. Included in A/S are spinning/non-spinning reserves and regulation services. Studies have shown that short-term A/S are the most beneficial use of automotive-type batteries 
[18], [21], [22], [27-30]. Because of the technical advantages that batteries have over traditional generation for $\mathrm{A} / \mathrm{S}$, such as fast response time, availability in small increments, their distributed nature, and ability to offset traditional generation and improve system efficiency, A/S is the most economically viable service for DES systems [12], [21], [22], [31]. Both capacity and energy payments are associated with A/S contracts; the generator is paid not only for energy transferred, but for being available for dispatch [7], [12]. Because DES systems are nearly always available, this capacity payment is often the main source of revenue for B2G [18], [32].

\subsubsection{SPINNING RESERVES}

The first type of $\mathrm{A} / \mathrm{S}$ considered are spinning and non-spinning reserves markets. The spinning reserve market specifies contracts for power capacity that is already synchronized to the grid to provide fast response (within 10 minutes of command). Non-spinning reserves are similar, although they are not required to be previously synchronized [15], [27]. Non-spinning reserves do not appear to be as profitable a market as spinning reserves, so they will not be discussed further. Spinning reserves are in operation only 10-20 times per year for 10 minutes to two hours [7], [18]. Batteries have been studied for this type of market because this low overall usage may not affect battery life considerably, batteries can provide faster response than typical reserves capacity, and synchronization to the grid is accomplished by simply plugging in to the wall, so the capacity payment does not incur costs [7], [22]. Analyses of spinning reserve feasibility have shown mixed results, however. A study by Cready et al. showed that costs incurred from spinning reserves were higher than potential revenue, while another study by Kempton et al. found most vehicle batteries would be profitable with spinning reserves [11], [22]. Moura concluded that EDVs are unappealing for spinning reserves, but Sioshansi et al. 
considered spinning reserves to be the most viable option [16], [31]. Either way, due to energy constraints, batteries become less useful as the contract period increases, so a market with even lower net energy would be more advantageous to B2G [18].

\subsubsection{FREQUENCY REGULATION}

The final A/S market for discussion is frequency regulation, which controls the minuteby-minute matching of power supply to instantaneous power demand [7]. Resources that currently provide regulation are conventional power plants and hydroelectric generators [27]. Because it is inherently a quick-response market (generators must respond to the command signal sent every 2-4 seconds), it is intuitive that a battery system would be a superior candidate for this market in particular [7], [11]. Furthermore, regulation services account for $80 \%$ of the A/S expenditures by the Independent System Operator (ISO); it is a much larger and more valuable market than spinning reserves [6], [7], [22]. Because the market is meant to control slight deviations (Fig. 1), the signal has been presumed to contain significantly less energy than the other markets and services, which is beneficial for the small energy capacities of batteries [18], [19], [22], [24], [26], [28]. Because frequency regulation has been demonstrated by many as the best choice of energy market for DES systems, my analysis is based on this market [7], [11], [12], [16], [18], [19], [22-24]. 


\subsection{Project Objectives and Research Challenges}

While low-power, low-energy distributed energy storage systems have the capability to meet the strategic goals of frequency regulation at low cost because of the low capital costs associated with smaller installations, the fast response available from smaller resources, and the availability of low-cost technologies including automotive-type batteries, there are still uncertainties as to some technical aspects of regulation for DES systems [12], [18]. The barriers to implementation of DES systems are the problems associated with system-level communication and control of a large number of distributed devices [30]. In order to realize the benefits of DES systems, the Balancing Authority (BA) needs to develop new methodologies for contracting and dispatching distributed storage systems. Recent work by Quinn et al. and Kempton et al. have shown that actuating DES using conventional systems leads to low reliability, low availability, and lower economic value [6], [33]. At present, many BAs and ISOs do not have a frequency regulation market specific to DES [19], [30]. The Automatic Generation Control (AGC) signal that is presently used for frequency regulation is a combination of Interchange Error, a frequency deviation term, an offset term for automatic time error correction, and an Hourly Inadvertent Energy Payback term. In general, these AGC components and the resulting AGC signal contain an overall energy bias [30], [33], [34]. The traditional AGC signal is tailored to the characteristics of traditional generators and will not be viable with energy-limited storage systems [6].

To enable the technical feasibility of storage systems for the electric grid, the ISO/BA will have to construct low-energy frequency regulation signals and a corresponding low-energy frequency regulation market product [35]. For instance, PJM has developed a fast-response, low net energy frequency regulation signal specifically to encourage the commercialization of DES. 
This new signal had a net energy of $\sim 1 \mathrm{MWh}$ in a period studied, whereas the conventional frequency regulation signal had a net energy of $\sim 40 \mathrm{MWh}[36]$. At present it is unclear what effect this net energy has on the reliability and function of DES, as described in my research questions:

RQ1. How can I characterize the acceptable amount of net energy on the AGC signal for DES?

RQ2. What capacities of DES will be able to participate in the PJM experimental market with reliability relative to production-market participants?

RQ3. What will be the characteristics of $\mathrm{A} / \mathrm{S}$ systems that can enable the participation of smallscale DES?

RQ4. What method of aggregation of the command signal should be used to maximize benefit for DES systems?

In order to quantify and compare the effects of these AGC signal characteristics on the reliability of DES systems as A/S providers, this study proposes a simplified computational model of the performance of DES resources. The results demonstrate the stochastic performance of these resources when actuated by synthetic AGC signals. Discussion focuses on evaluation of methods to improve DES system reliability, synthesis of system reliability under varying AGC signal conditions, and assessment of the currently available AGC signals within the proposed modeling framework. 


\subsection{Models ANd Methods}

A DES system is comprised of numerous ES devices, and the reliability of the whole can be deduced from the reliability of each device. This model consists of a simple representation of an ES device subject to a power command (representing a portion of the AGC signal). The ES device attempts to meet the power command to the best of its capability. For simplicity, the ES device is assumed to have $100 \%$ coulombic and energetic efficiency, such that the device energy content can be calculated from the integrated power command. The ability of the ES device to meet the power command is limited only by the energy capacity of the device; the current and voltage that might be required at any given power are assumed to be within the physical and electrochemical limits of each device.

The power command to each ES device is assumed to be a component of the area-wide AGC signal. For this study, the AGC signal is assumed to be a sinusoidal signal of constant period, constant amplitude, and zero offset. There are two possible means for distributing this high-power $\left(\mathrm{P}_{\text {signal }}\right)$ signal to the low-power $\left(\mathrm{P}_{\text {storage }}\right)$ ES units. The stacked call method (Fig. 2) divides the high power signal into a number of quantized signals with a power of $\mathrm{P}_{\text {storage. }}$. Commands issued to the individual ES devices can only have values of $\pm \mathrm{P}_{\text {storage }}$ or zero. The power outputs are summed from numerous distributed ES devices to meet the total power command. Alternatively, the proportional call method (Fig. 3), as used by PJM Interconnect (PJM) and the California ISO (CAISO), divides the high power signal into a number of continuous, time-varying signals [24], [36]. Each storage device is commanded with a constant fraction of the instantaneous AGC. To model these two control strategies, the power command to the ES device is modeled as either a square wave or a sinusoidal wave. Both commands are characterized by their period and peak amplitude. 


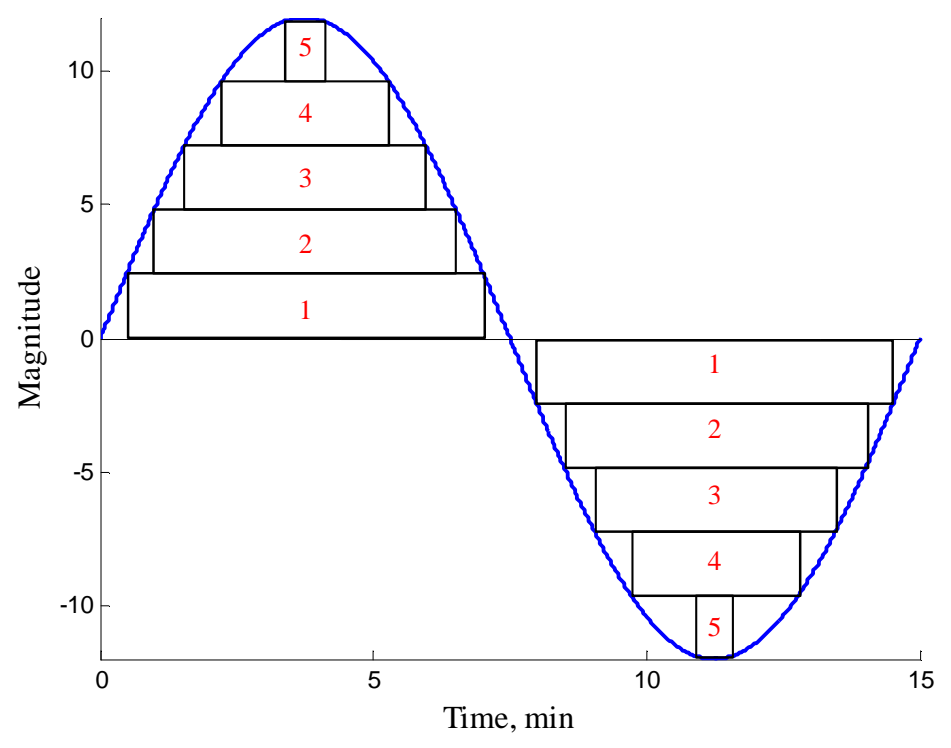

Fig. 2: Stacked call method for DES signal distribution

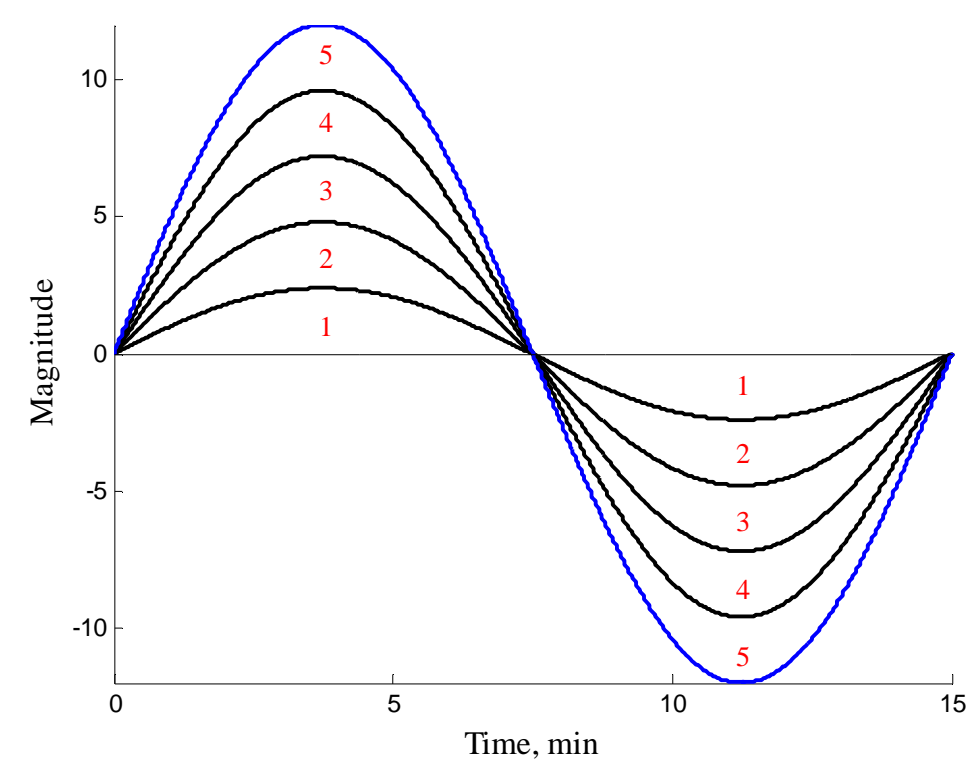

Fig. 3: Proportional call method for DES signal distribution

$\mathrm{A} / \mathrm{S}$ are assumed to be procured by the BA using a series of 10 minute contract periods which matches the dispatch period used by CAISO [37]. During this contract period, each 
device can perform either regulation down (drawing power from the grid) or regulation up (sending power to the grid), based on the command signal.

\subsection{NZETRATIO DEFINITION}

In this study, I characterize the power command by the parameter Net-Zero-Energy Time (NZET). NZET is the time at which the command signal has requested equal amounts positive and negative energy. In this model, where symmetric, periodic signals are used, NZET is equivalent to the period of the power command. NZET can be normalized by the capacity of the ES device $\left(\mathrm{Q}_{\mathrm{ES}}\right)$ to calculate NZETRatio, a dimensionless parameter defined in (1). This ratio is used because it not only accounts for the effects of the grid signal (power and period), but also the energy storage capability of the ES device. By using the ratio as opposed to strict NZET, the simulation results are non-dimensional in order to show ES device performance independent of scale.

In order to establish the basis for the NZETRatio, I arbitrarily assigned an ES device size to the model. For ease of calculation and generation of results, $\mathrm{P}_{\text {signal }}=12 \mathrm{~kW}$ and $\mathrm{Q}_{\mathrm{ES}}=1 \mathrm{kWh}$. Using (1), for NZETRatio $=1$, NZET $=1 / 12$ hour $=5$ min. For a NZETRatio of 1 an ES device with an initial state-of-charge (SOC) of zero will charge to $50 \%$, then return to zero in one netzero-energy period, as shown in Fig. 4.

NZETRatio $=\frac{\mathrm{P}_{\text {signal }} * \text { NZET }}{\mathrm{Q}_{\mathrm{ES}}}$ 


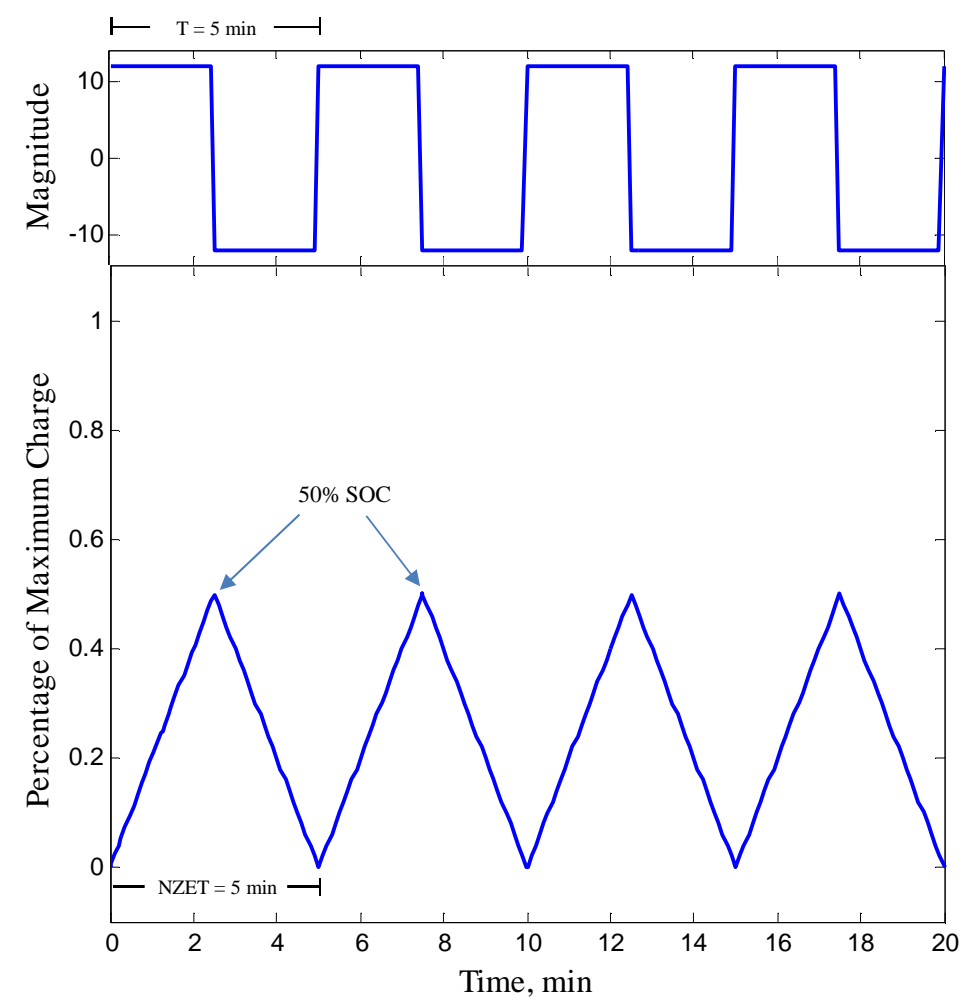

Fig. 4: SOC of ES device over time with NZETRatio $=1$ and $100 \%$ call ratio, with the grid command signal above

\subsection{RELIABILITY DEFINITION}

Reliability in this study is calculated so as to be analogous to the ratio of NERC reported Equivalent Forced Derated Hours (EFDH) and Service Hours (SH) as defined in (2) [38].

$$
\mathrm{R}=1-\frac{\mathrm{EFDH}}{\mathrm{SH}}
$$

For my purposes, the EFDH is the time at which the ES device is contracted to provide regulation but is unable to transfer energy (due to a SOC of $0 \%$ or $100 \%$ for regulation up or down, respectively). $\mathrm{SH}$ is the total time a device is under contract to provide regulation. 
The steady-state reliability of the system is simulated by removing the first 30 minutes (three contract periods) of each simulation from the reliability calculation. This ensures that the simulation is at steady-state before its reliability is assessed.

\subsection{CALl RATio DEFinition}

Call ratio is defined as the ratio of $\mathrm{SH}$ (the time during which the resource is providing energy) to available hours ( $\mathrm{AH}$, the time during which the resource is available for contracting). In my model, this is a stochastic factor based on how often the aggregated ES devices are commanded to provide energy services. For example, a power command with $50 \%$ call ratio will allow the DES system to participate in a random time series of $50 \%$ of the contract periods. Studies of distributed energy services to date have assumed 10\% call ratio (based on the fraction of required $\mathrm{A} / \mathrm{S}$ power to contracted $\mathrm{A} / \mathrm{S}$ resources), but NERC reports that pumped hydroelectric generators can approach 70\% call ratio [18], [23], [38]. Because fast-response DES systems provide high-value service to the ISO, high call ratios are likely to result [33].

\subsection{DeVice-LeVel State of Charge Management}

One method used by this simulation manages SOC at the device level using non-contract charging, wherein when not dispatched to provide regulation services, each ES device charges or discharges to achieve 50\% SOC. This would be accomplished by switching between regulation markets when contracted and the energy market when not contracted. By employing a noncontracted period to charge to $50 \%$, the reliability of the fleet increases due to the ability for each ES device to provide for either regulation up or down, as opposed to providing just one or the 
other due to incurring a saturation limit [11], [12], [33]. This device-level SOC management will be applied to the simple symmetric market (see section 7.0).

\subsection{A/S MARKET STRUCTURE}

There are two A/S regulation markets considered within which to bid in this study: a symmetric structure, where participation in the market requires one symmetric regulation up and regulation down contract (used by PJM [7]), and an asymmetric structure, wherein an A/S provider may bid into either regulation up or regulation down markets or both (used by CAISO [37]). The latter gets its name because of the decoupling of the bidding capacity of the ES device for each market (i.e. $1 \mathrm{MW}$ regulation up and $1.5 \mathrm{MW}$ regulation down would be valid).

The asymmetric bidding is controlled for each ES device based on the SOC at the beginning of each contract period. If the SOC is above the upper control point, the ES device will participate in only the regulation up market (device discharging). Similarly, an SOC below the lower control point results in only regulation down contracts (device charging).

Control points for this simulation were chosen to maximize the reliability over the largest possible NZETRatio range. A study was run to determine which of five pairs of control points would provide the highest reliability (see section 12.1). Because optimization of these control points is beyond the scope of this paper, all pairs used in this study were symmetric about $50 \%$ SOC. $100 \%-0 \%$ control with a $0.5 \%$ offset to allow for numerical error (where $99.5 \%$ SOC is the upper control point and $0.5 \%$ SOC is the lower) was shown to provide one of the most reliable DES in the range NZETRatio $\leq 2$ and the highest DES reliability for $2<$ NZETRatio $\leq 4$ by a point-to-point minimum of $9.0 \%$ with an average increase in reliability across all points in this range of $15.0 \%$ for square-wave input. The maximum simulated command signal period is 
NZETRatio $=4$ because reliability here is too low to be of use and the general trend of each curve is understandable over this range.

Results under the symmetric market structure will be displayed as-is to show current PJM markets with no modifications, as well as paired with non-contract charging to show the effects of bidding into A/S markets alternatively with the energy market. The asymmetric structure will also be presented as an example of CAISO markets and as another method with which to increase DES system reliability.

\subsection{COMPUTATIONAL METHODS}

The individual ES device is Monte Carlo simulated using a constant distribution of initial device SOC. A sample size of $n=200$ was used. To reach this sample size, I ran a characteristic study of reliability for the Monte Carlo method at NZETRatio $=5$. This NZETRatio was chosen because that point has a consistently low reliability and therefore has a low probability of a string of simulations achieving $100 \%$ reliability and skewing the results. Also, the NZET is 25 minutes in this case, which allows for a sufficient number of contract periods to promote high confidence in the calculated reliability. The error in reliability of the 100 -sample case is within $0.004 \%$ of the 10,000-sample case (Table 1). I therefore concluded that 200 samples are enough to quantify stochastic reliability.

Table 1: Reliability of a selection of sample sizes for NZETRatio = 5

\begin{tabular}{|c|c|}
\hline Sample Size (n) & Reliability \\
\hline 20 & 0.4319 \\
\hline 100 & 0.4316 \\
\hline 1000 & 0.4288 \\
\hline 10000 & 0.4299 \\
\hline
\end{tabular}


The output of the simulation is the expected value of the stochastic reliability for a single ES device as a function of market structure, call ratio, and NZETRatio.

\subsection{ANALYTICAL SOLUTION}

To validate the simulation results, I developed an analytical solution for each distribution method at $100 \%$ call ratio. Equation (3) relates the energy of the command signal over the reliable time of the ES device to the storage capacity of the device.

$\int_{0}^{\mathrm{t} \_ \text {deplete }} \mathrm{CS} d t=\mathrm{Q}_{\mathrm{ES}}$

where CS is the power command signal. The variable $t_{\text {deplete }}$ represents the time at which an ES device can no longer transfer energy for the current command signal request and therefore is no longer reliable. Furthermore, the ratio of $\mathrm{t}_{\text {deplete }}$ to contracted time is the reliability of the ES device for that contract.

This is related to reliability through (2) in which the EFDH is the time remaining in onehalf the command signal NZET after $t_{\text {deplete }}$ occurs (4a).

$$
\mathrm{R}=\frac{\mathrm{SH}-\mathrm{EFDH}}{\mathrm{SH}}=\frac{2 \mathrm{t}_{\text {deplete }}}{\mathrm{NZET}}
$$

Reliability is then related to NZETRatio by incorporating the signal and ES device components directly (4). 
$\mathrm{R}=\frac{2 \mathrm{P}_{\text {signal }} * \mathrm{t}_{\text {deplete }}}{\text { NZETRatio } * \mathrm{Q}_{\mathrm{ES}}}$

\subsubsection{STACKED CALL AGC DISTRIBUTION}

The energy of the square waveform can be calculated by integrating the Heaviside function $\mathrm{H}(\mathrm{t})$ for a full net-zero-energy period (5-5a). A net-zero-energy period is represented by a through $\mathrm{c}$, where for the first period, $\mathrm{a}=0, \mathrm{~b}=$ NZET/2, and $\mathrm{c}=$ NZET (Fig. 5).

$\mathrm{CS}=\mathrm{P}_{\text {signal }}\left(\mathrm{H}_{\mathrm{ab}}+\left(-\mathrm{H}_{\mathrm{bc}}\right)\right)$

where

$\begin{cases}\mathrm{H}_{\mathrm{ab}}=1 & \text { if } \mathrm{a}<\mathrm{t} \leq \mathrm{b} \\ \mathrm{H}_{\mathrm{bc}}=1 & \text { if } \mathrm{b}<\mathrm{t} \leq \mathrm{c}\end{cases}$

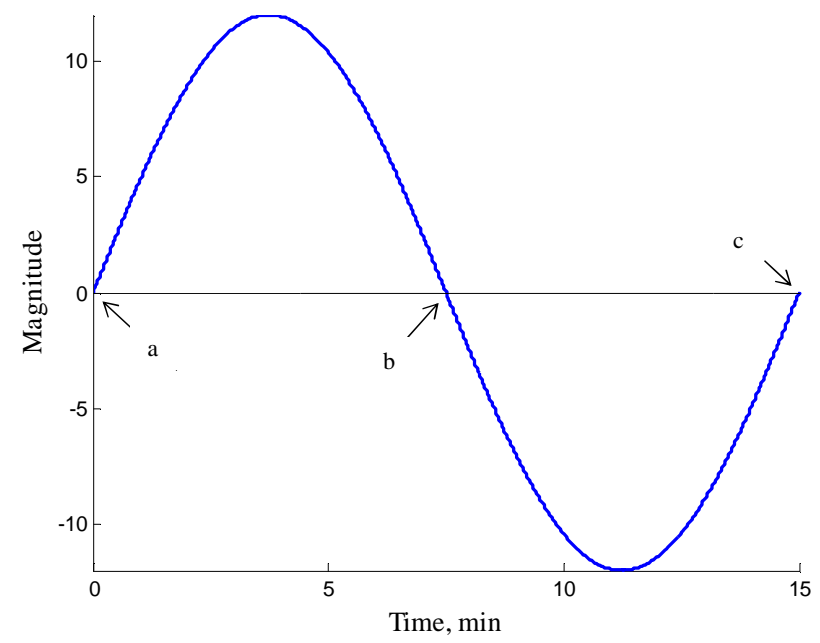

Fig. 5: Position of values $a, b$, and $\mathbf{c}$ for the first net-zero-energy period of a power command signal where

$$
\text { NZET }=15 \mathrm{~min}
$$


Integration of the Heaviside function with respect to the initial conditions (6) results in the ramp function $\mathrm{R}(\mathrm{t})$ (7-7a).

$$
\begin{gathered}
\left\{\begin{array}{c}
\mathrm{E}_{\text {signal }}(\mathrm{a})=0 \\
\mathrm{E}_{\text {signal }}(\mathrm{b})=\mathrm{P}_{\text {signal }} *(\mathrm{~b}-\mathrm{a})
\end{array}\right. \\
\mathrm{E}_{\text {signal }}=\mathrm{P}_{\text {signal }}\left(\mathrm{R}_{\mathrm{ab}}+\mathrm{R}_{\mathrm{bc}}\right)
\end{gathered}
$$

where

$$
\left\{\begin{array}{cc}
\mathrm{R}_{\mathrm{ab}}=\int \mathrm{H}_{\mathrm{ab}} \mathrm{dt}=\mathrm{t}-\mathrm{a} & \text { if } \mathrm{a}<\mathrm{t} \leq \mathrm{b} \\
\mathrm{R}_{\mathrm{bc}}=\int \mathrm{H}_{\mathrm{bc}} \mathrm{dt}=2 \mathrm{~b}-\mathrm{a}-\mathrm{t} & \text { if } \mathrm{b}<\mathrm{t} \leq \mathrm{c}
\end{array}\right.
$$

From (7), the time at which $\mathrm{E}_{\text {signal }}=\mathrm{QES}_{\mathrm{ES}}\left(\mathrm{t}_{\text {deplete }}\right)$ was calculated and entered into (4).

\subsubsection{PROPORTIONAL CALL AGC DiSTRIBUTION}

The sinusoidal command signal energy content was able to be calculated directly from (3), with the results shown in (8-9a).

$E_{\text {signal }}=\frac{N Z E T * P_{\text {signal }}\left(1-\cos \left(\frac{2 \pi t}{\text { NZET }}\right)\right)}{2 \pi}$ 
Solving for $\mathrm{E}_{\mathrm{signal}}=\mathrm{Q}_{\mathrm{ES}}$ gives

$$
t_{\text {deplete }}=\frac{-\operatorname{NZET}\left(2 \sin ^{-1} x-\pi\right)}{4 \pi}
$$

where

$$
\mathrm{x}=\frac{\mathrm{NZET} * \mathrm{P}_{\text {signal }}-2 \pi \mathrm{Q}_{\mathrm{ES}}}{\mathrm{NZET} * \mathrm{P}_{\text {signal }}}
$$

\subsubsection{ANALYTICAL RESULTS}

Shown in Fig. 6 are the results of the analytical solution for both sinusoidal and square wave command signals. These results will be shown to correspond directly with the $100 \%$ call ratio results from the symmetric simulations. Call ratios below $100 \%$ are not addressed in the analytical solution because the stochastic effects of lower call ratios necessitate numerical simulation. 


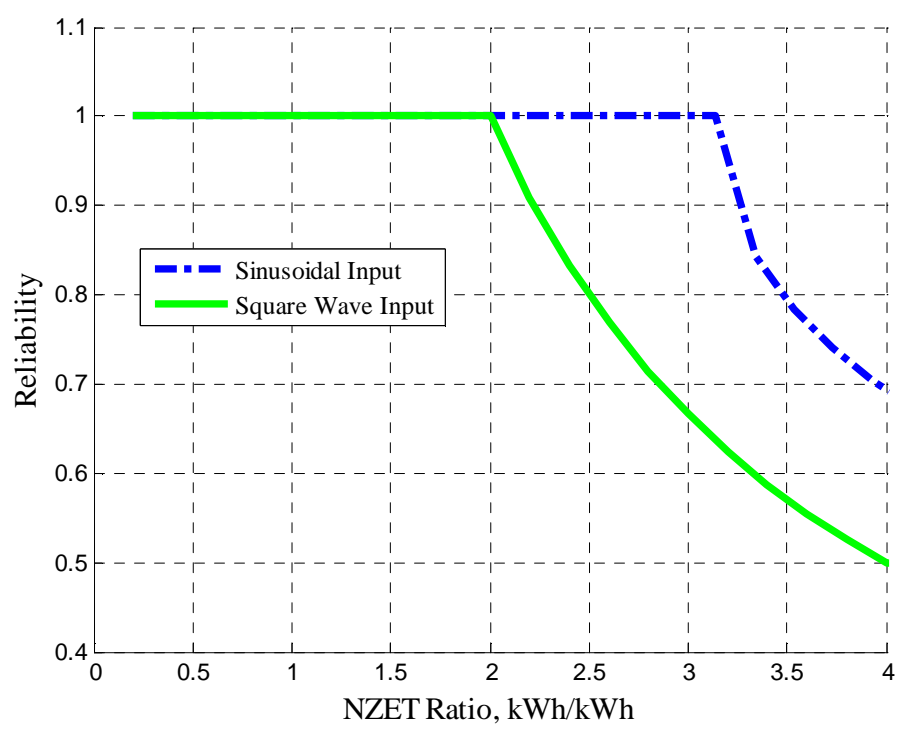

Fig. 6: Analytical solution to reliability of DES at $100 \%$ call ratio

The NZETRatio at which the reliability curve begins to decline is labeled as the breakpoint. This breakpoint occurs at NZETRatio $=2$ for a square-wave input (when the ES device capacity is equal to the command signal energy) and NZETRatio $=\pi$ for a sinusoidal input. This can be easily explained by computing the energy of a sinusoid over half its period and comparing it to that of the square-wave with the same half-period. The sinusoid of the same peak power contains lower energy content than the square wave by a factor of $\pi / 2$; therefore, the curves are shifted by this factor and the reliability for a given sinusoidal NZETRatio is higher.

\subsection{LIMITATIONS}

This model and the accompanying analyses are meant to provide guidelines for future development, not to develop specific results. The author realizes that certain parameters are not necessarily as they would be in real-world situations. Constant call ratio simulations serve to show the reliability trend, even though call ratio is not actually constant for extended periods. 
This model uses sinusoidal waves for the proportional call; I realize an AGC signal will not be that smooth, but sinusoids provide a good comparison to the stacked-call method at most points. Also, I refrain from studying specific NZETRatios in detail because ranges are more pertinent to a constantly-varying real-world AGC signal.

Even though points below approximately $95 \%$ reliability are unusable to the ISO (NERC reports a representative reliability of gas turbines to be $98.89 \%$ [33]), I feel it is important to show the overall trend. In these ways, the results can be instructive about methods to increase reliability of DES systems, not simply output strict imperatives. 


\subsection{Results for Simple Symmetric STRUCture}

\subsection{RESUlTS WITH SQUARE-WAVE INPUT}

The following sections discuss the reliability results of the simulated ES device with varying NZETRatio and call ratio. Fig. 7 shows a plot of the ES average reliability over time for four values of call ratio: $10 \%, 50 \%, 80 \%$, and $100 \%$. Two areas of specific interest are marked on the plot and will be discussed in detail.

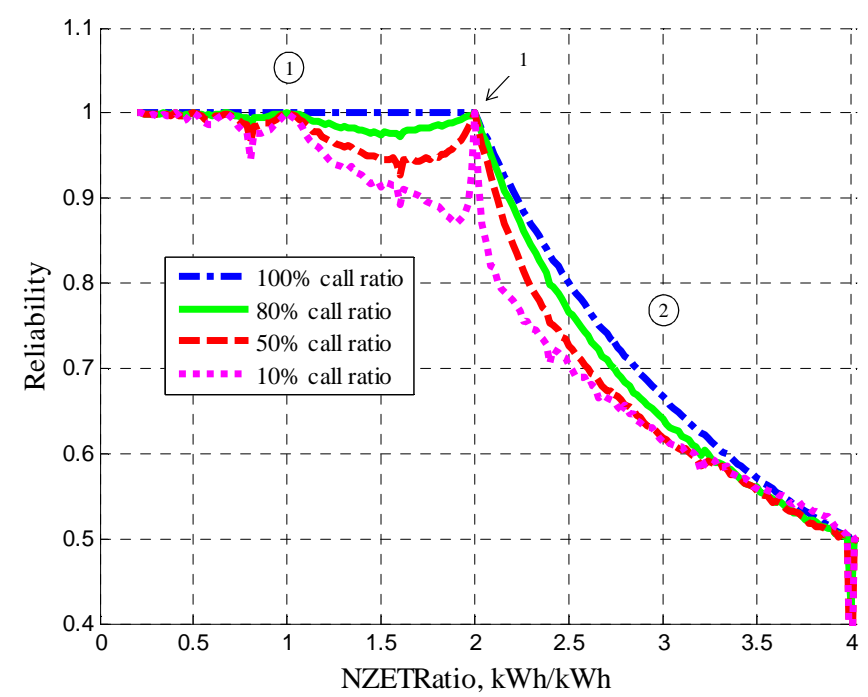

Fig. 7: Reliability vs. NZETRatio for three call ratio values and square-wave input and simple symmetric market 


\subsubsection{NZETRATIO $\leq 2$}

For any call ratio through NZETRatio $=1$, ES devices are very reliable $(>97 \%$ for most NZETRatios). The $100 \%$ call ratio is $100 \%$ reliable in this range, while the other call ratios display predictable deviations as described in section 12.2 (Fig. $8^{1}$ ). After the transient period is filtered out, the $100 \%$ call ratio ES device will be in either the $0-50 \%$ SOC range or the $50-100 \%$ SOC range because this range of NZETRatio is defined as less than $50 \%$ use of the DES. During the deviations, lower call ratio curves show slight dips in reliability, increasing with decreased call ratio. This is because the lower the call ratio, the higher the probability of the ES device being commanded two or more consecutive calls with the same sign (i.e. up, up). Signals with NZETRatio $\leq 1$ are preferable for energy-limited storage systems because all ES devices are near $100 \%$ reliable regardless of call ratio.

\footnotetext{
${ }^{1}$ For result plots in sections 6.0 and 8.0, predictable deviations occur in the range NZETRatio $\leq 2$. This is due to the relationship between energy input to and output from the ES device. Unlike single-point increases or decreases in reliability, these macroscopic deviations are indicative of real-world reliability variations. For more information on both types of deviation, see section 12.2.
} 


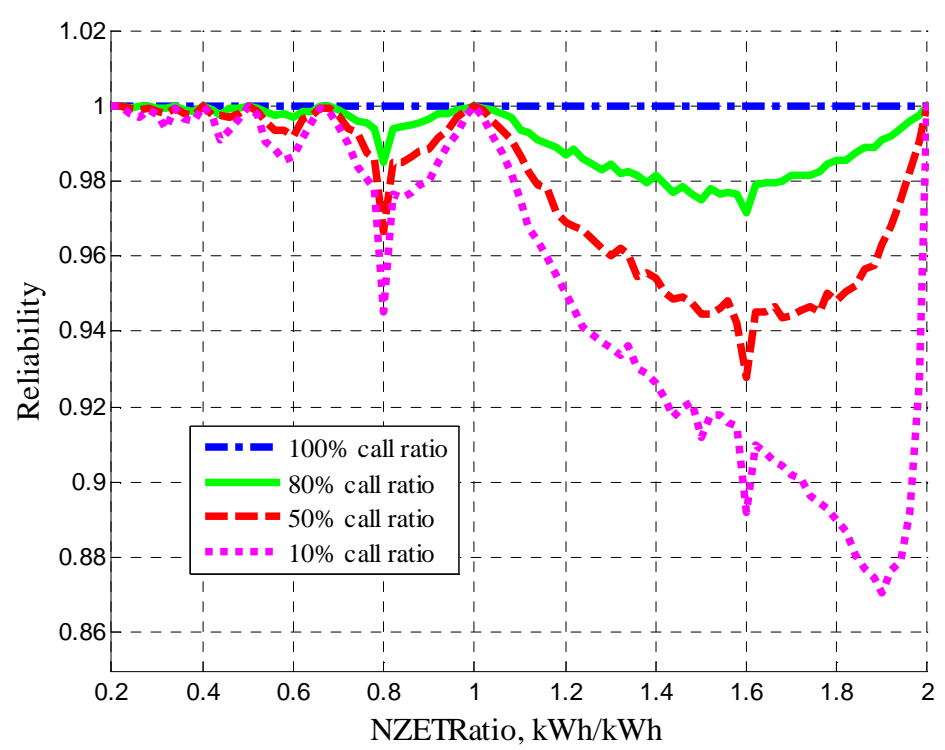

Fig. 8: Reliability vs. NZETRatio for three call ratios and square-wave input with simple symmetric market for NZETRatio $\leq 2$

In the range $1<$ NZETRatio $<2$, the difference in call ratio has more effect. For the $100 \%$ call ratio scenario, ES device reliability remains at 1 for all NZETRatios $\leq 2$. Resource reliability again decreases with call ratio in a predictable fashion that is more dramatic in this range. The $100 \%$ call ratio curve maintains a reliability of 1 because there are no non-contracted periods; the ES device will oscillate between $0 \%$ and 100\% SOC. Fig. 9 shows that after the initial transient period, the ES device does indeed oscillate exactly between the saturations limits for NZETRatio $=2$. 


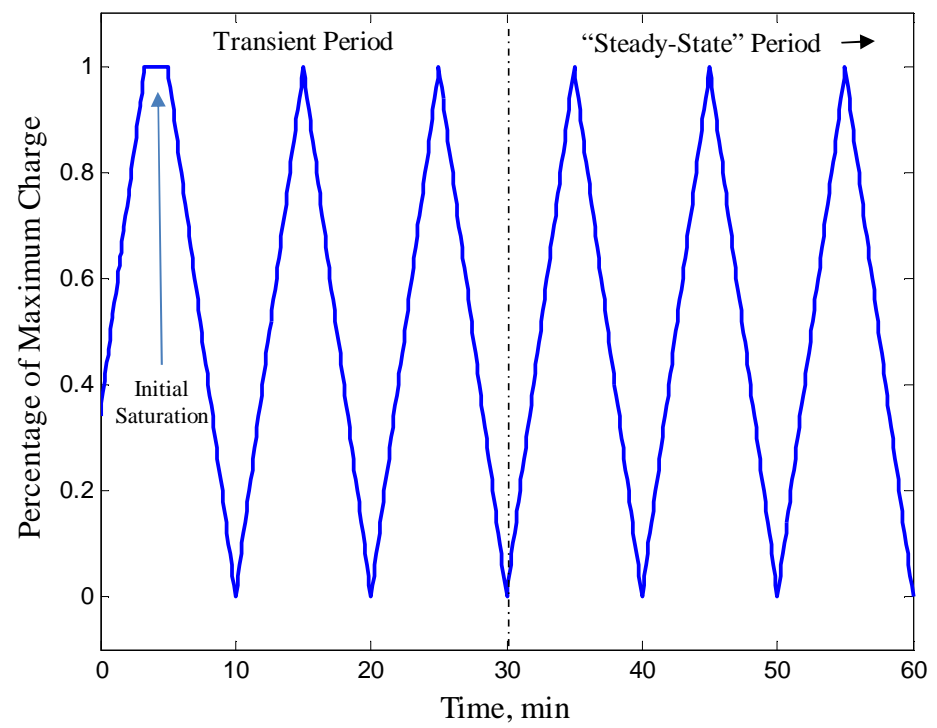

Fig. 9: SOC over time with $100 \%$ call ratio and initial saturation in the disregarded transient period at NZETRatio $=2$

\section{$6.1 .22<$ NZETRATIO $<4$}

For DES, the lower the call ratio, the lower the reliability not only for NZETRatio $<2$, but the entire range $2<$ NZETRatio $<4$ due to the probability of consecutive same-sign calls (NZETRatio $=2$ is covered in section 12.2). In this range, the reliability difference between the maximum and minimum curves decreases as NZETRatio increases. This is because as command signal NZET increases relative to the energy capacity of the ES device, consecutive contracted periods become less valuable; the oscillation in the command signal is slow enough that saturation will be incurred regardless. This is also the reason for the decrease in the reliability difference between call ratios over this range to a maximum of $0.11 \%$ for NZETRatio $=4$.

\subsection{RESULTS WITH SINUSOIDAL INPUT}

The reliability curve for the NZET sweep with sinusoidal input (Fig. 10) has a similar shape to the results from section 6.1 with some key differences. The predictable deviations 
mentioned above are not shifted in the same fashion as the breakpoints. This is because the deviations are associated with the length of the contract period rather than command signal energy content (more in section 12.2).

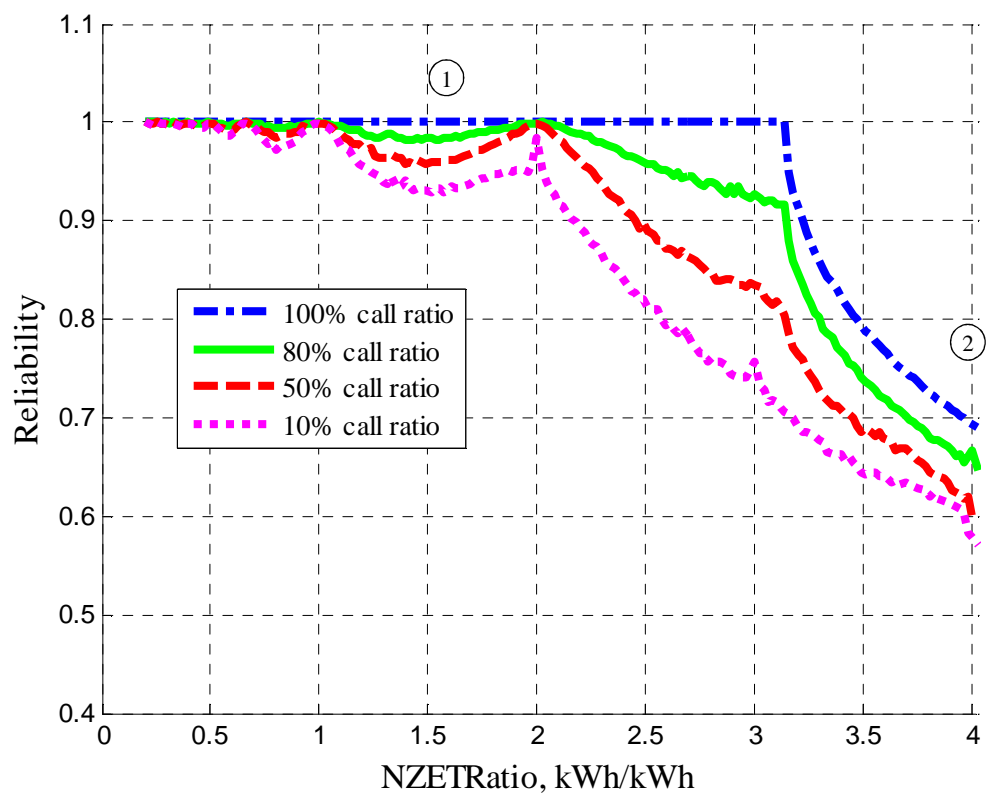

Fig. 10: Reliability vs. NZETRatio for three call ratio values and sinusoidal input with simple symmetric market

The main difference is associated with the difference in energy between the two command signals. There is a smaller reliability difference between maximum and minimum with sinusoidal input for equivalent NZETRatios. For range (1) (NZETRatio $\leq 2$ ), the sinusoidal command signal has a maximum reliability deviation between the studied call ratios of $7.1 \%$ (occurring at NZETRatio $=1.54$ ) while the square-wave command signal has a maximum of $13 \%$ occurring at NZETRatio $=1.90$. This large difference in maximum deviation shows that a sinusoidal command signal will provide overall higher reliability with an equivalent call ratio in this range. 
In range (2), the opposite is true regarding reliability difference. The square-wave command signal reliability displays a much narrower overall difference, with a maximum deviation between call ratios of $14 \%$ at NZETRatio $=2.14$ while the sinusoidal input has a maximum deviation of $29 \%$ at NZETRatio $=3.20$. The trend shows that for NZETRatio $>2$ a decrease in call ratio has a larger effect on reliability with a sinusoidal input than with squarewave. While the resource reliability difference is much greater, all of the studied call ratios with sinusoidal input have greater reliability than the maximum square-wave reliability over nearly the entire range (2) (Fig. 11). Therefore, a sinusoidal command signal provides higher reliability in this range as well.

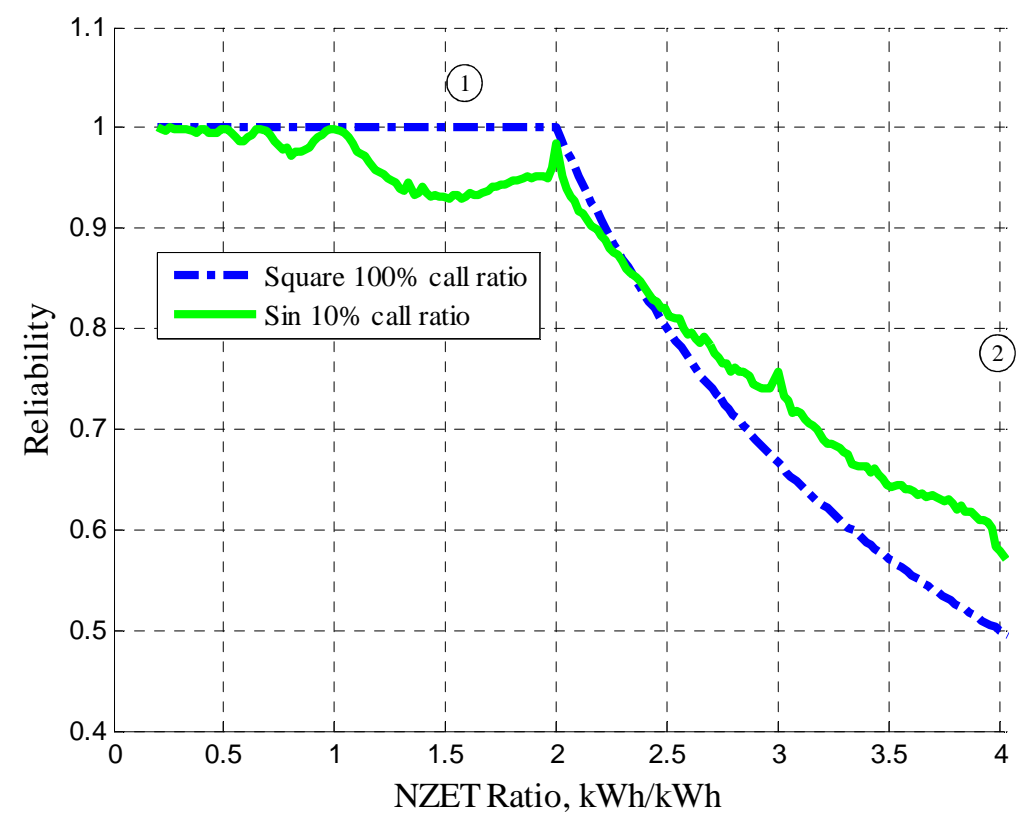

Fig. 11: Comparison of maximum square-wave reliability with minimum sinusoidal reliability for simple symmetric structure 


\subsection{RESUlts For Symmetric STRUCTURE With SOC MANagement}

\subsection{RESUlTS WITH SQUARE-WAVE INPUT}

The following sections discuss the reliability results of the simulated ES device with varying NZETRatio and call ratio. Fig. 12 shows a plot of the ES device average reliability over time for four values of call ratio: $10 \%, 50 \%, 80 \%$, and $100 \%$.

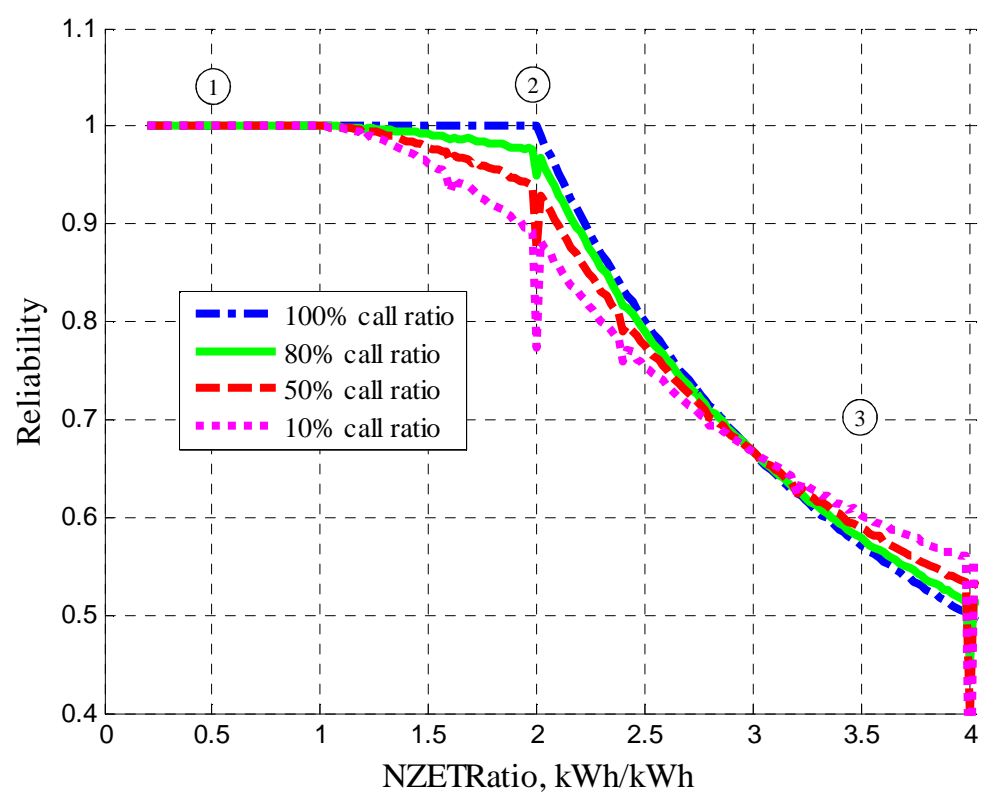

Fig. 12: Reliability vs. NZETRatio for three call ratio values and square-wave input with SOC management

The difference in simulation structure between this section and the previous is that here results are shown with the same DES and market structure but with non-contract charging applied. For example, the $100 \%$ call ratio curve is identical to that of section 6.0 because noncontract charging is not applicable. 


\subsubsection{NZETRATIO $\leq 1$}

All ES devices for any call ratio through NZETRatio $=1$ are $100 \%$ reliable. Noncontract charging is responsible for the lack of predictable reliability deviation seen in the previous section. This is the range of $\leq 50 \%$ use of the device, so by applying SOC management that dis/charges the device to $50 \%$ SOC during non-contracted periods, a saturation limit will never be reached. Signals with NZETRatio $\leq 1$ are preferable for energy-limited storage systems with SOC management because reliability is $100 \%$ and independent of call ratio.

\subsection{2 $1<$ NZETRATIO $\leq 3$}

For ES devices, the lower the call ratio, the lower the reliability not only for NZETRatio $=2$ (unlike section 6.0), but the entire range $1<$ NZETRatio $<3$. The main difference between this and the simple system (section 6.1) is that each curve with SOC management has a smoother profile. This is because when the SOC management "resets" each device to 50\% SOC during each non-contracted period, it is more probable that the ES device will have reliability similar to other samples in the simulation.

\subsubsection{NZETRATIO > 3}

This range is different than that without SOC management: for NZETRatio > 3, a lower call ratio results in greater reliability. This is a function of large NZET and dis/charging to $50 \%$ SOC. Because the NZET is large, there will be consecutive contract periods with the same command. When the SOC of a device is "reset" to $50 \%$, that device can then respond to a command for which it had previously been saturated. As NZET increases, SOC management 
continues to increase the reliability of the call ratios under 100\%. For NZETRatio $>3$, lower call ratio improves the reliability of the DES.

\subsection{RESULTS WITH SINUSOIDAL INPUT}

The sinusoidal command signal results are shown in Fig. 13. They are qualitatively similar to those of the square-wave input in that all call ratios are $100 \%$ reliable halfway to the breakpoint, then lower call ratios result in lower reliability. All points are significantly more reliable than the corresponding square-wave points. These results are also similar to the sinusoidal command signal of section 6.2 , barring the predictable deviations not seen here.

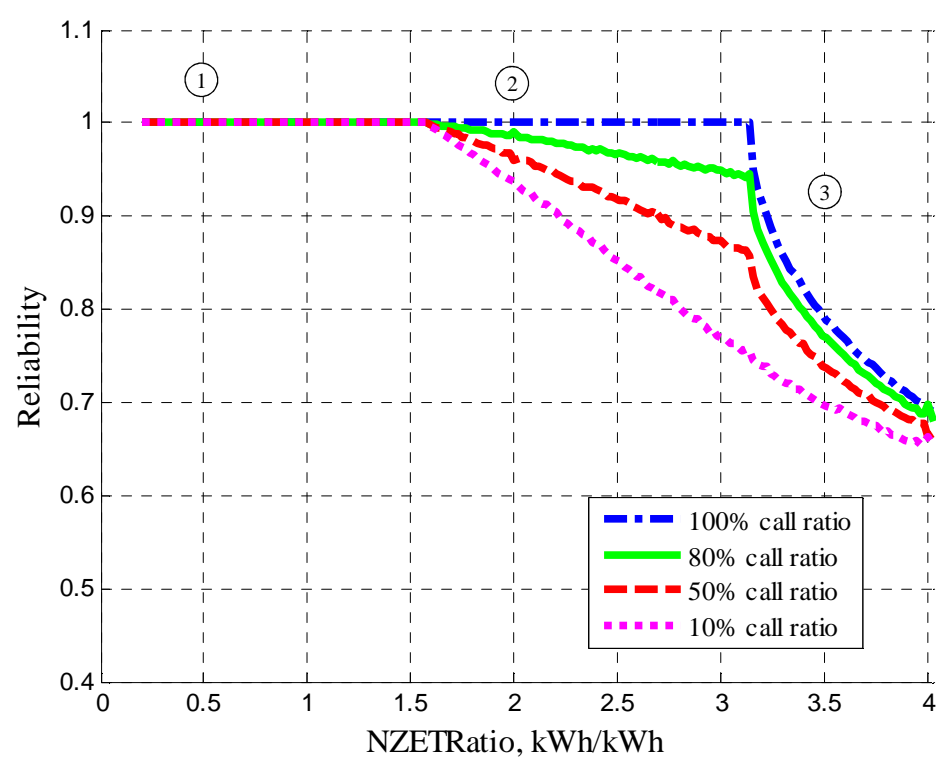

Fig. 13: Reliability vs. NZETRatio for three call ratio values and sinusoidal input with SOC management

The clear difference between the two sinusoidal results is that the SOC management smoothes the predictable declines in reliability for NZETRatio $\leq 2$. This dis/charge to $50 \%$ SOC 
has the effect of increasing the reliability of all points in this range, except for the rise to NZETRatio $=2$.

The reliability at each point for NZETRatio $>2$ also is slightly increased by SOC management. For $80 \%$ call ratio, the reliability with SOC management is an average $1.7 \%$ greater than without SOC management. Unlike the square-wave command signal with SOC management, there is no range studied with the sinusoidal command signal in which lower call ratios produce greater reliability. The similarities between these results and those of the simple symmetric sinusoidal results of section 6.2 warrant the same general conclusion be drawn: if using SOC management, a sinusoidal command signal provides better resource reliability for all ranges studied. 


\subsection{ReSUlts FOR ASYMmetric Structure}

\subsection{RESUlTS WITH SQUARE-WAVE INPUT}

The following sections discuss the resource reliability results of the simulation with varying NZETRatio and call ratio with a different A/S structure (Fig. 14). Through the asymmetric structure I will discuss key points of the B2G average reliability. Two areas of specific interest are marked on the plot and will be discussed in detail.

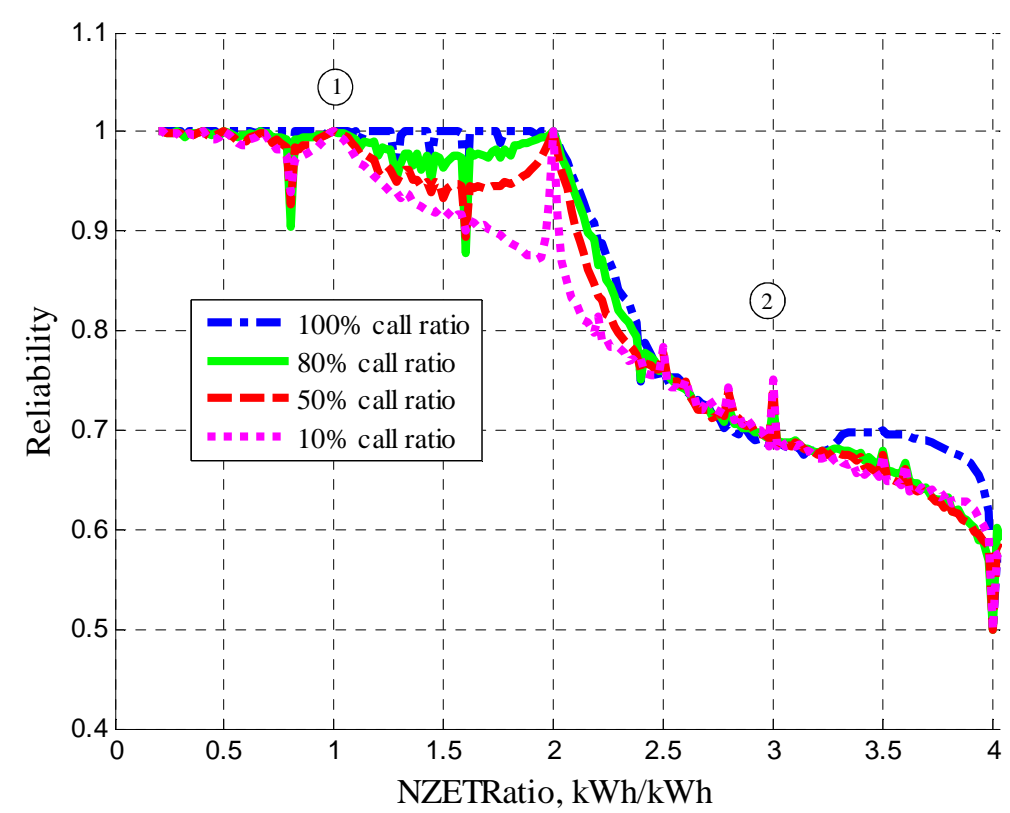

Fig. 14: Reliability vs. NZETRatio for four call ratios and square-wave input with asymmetric market

\subsubsection{NZETRATIO $\leq 2$}

This region is nearly identical to the symmetric structure without SOC management (section 6.1), including expected deviations. The coefficients of determination between the two curves are $0.83,0.88,0.86$, and 0.84 for the $100 \%$ and descending call ratios, respectively. Fig. 15 shows a graphical comparison of the two market structures for $10 \%$ call ratio. Accounting for the random nature of all results and the slight noise in the asymmetric data due to added 
complexity and rigid control points, these coefficients present very similar results between this and the simple symmetric structure in this range. The identical nature of the two plots has to do with the small NZET in this range and the strict control used for the asymmetric structure. Because the NZETRatio is small, the ES device can easily manage the energy content of the command signal and the probability of a saturation limit incurring is low. My control strategy for the asymmetric structure is for the device to bid in one market only if a saturation limit is nearly encountered; very few are encountered, so the results are nearly identical.

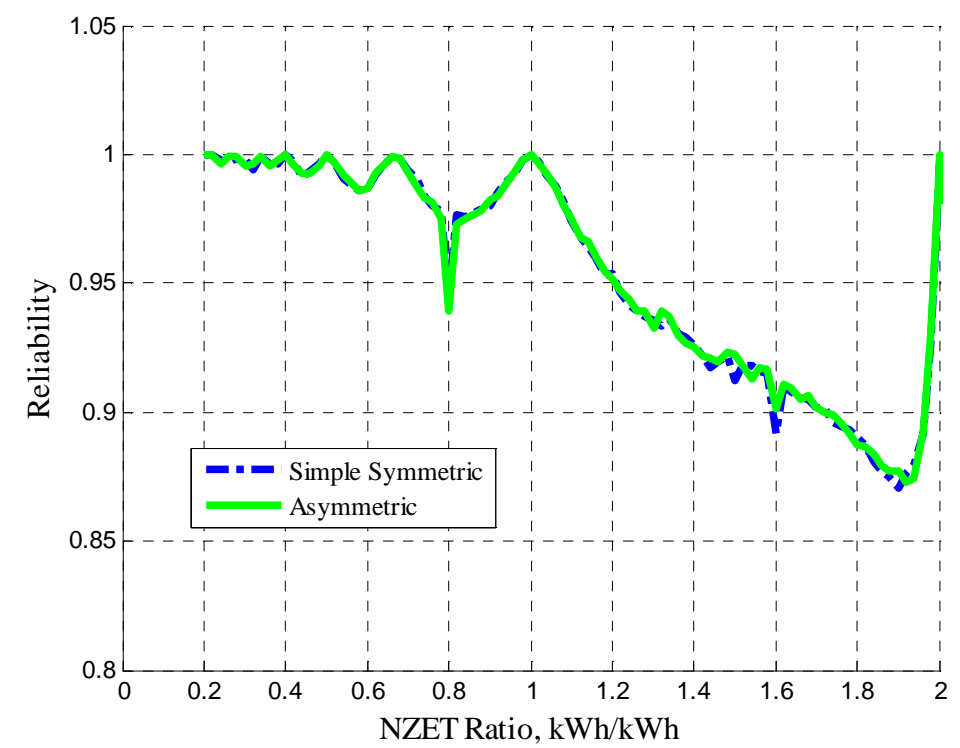

Fig. 15: Comparison of simple symmetric market and asymmetric market for square-wave input at $10 \%$ call ratio

\subsection{2 $2<$ NZETRATIO $\leq 4$}

Just after the breakpoint, the asymmetric results show mixed improvements and declines relative to section 6.1. For any call ratio larger than 50\%, the asymmetric structure is less reliable than the simple symmetric structure immediately after the breakpoint (Fig. 16). This 
length and depth of this reliability dip are inversely related to the call ratio; the $100 \%$ call ratio has a maximum relative reliability drop of $5.7 \%, 80 \%$ call ratio is $2.4 \%$, and the $50 \%$ call ratio relative reliability drop is $0.96 \%$. For the next call ratio studied, $10 \%$, the relative drop is an insignificant $0.24 \%$. After the short reliability drop for $2 \leq$ NZETRatio $\leq$ no greater than 3 , the asymmetric structure shows dramatic increases in reliability compared to the simple symmetric structure, with a maximum of $12 \%$ for every call ratio (Fig. 16).

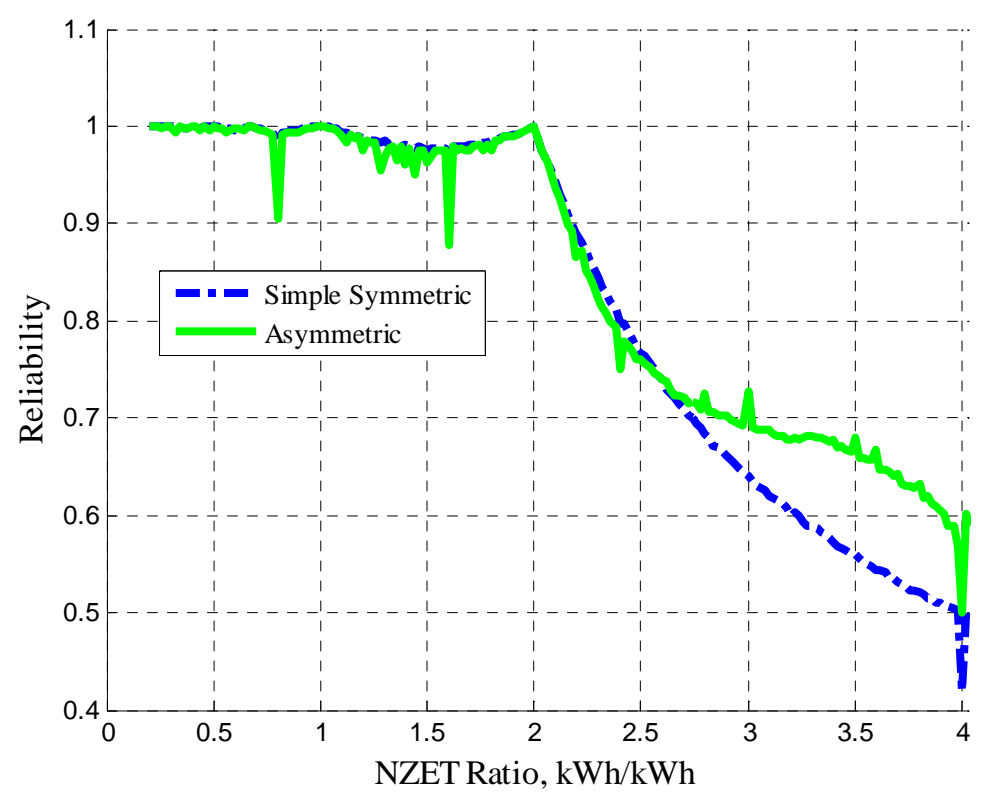

Fig. 16: Reliability vs. NZETRatio for $80 \%$ call ratio and square-wave input with simple symmetric and asymmetric markets

The asymmetric structure performs as expected when compared with the single market structure with SOC management described in section 7.1, considering the comparison between sections 6.1 and 7.1. The dual market structure has more in common with the results of section 6.1 than of those with SOC management. 
An advantage of the asymmetric structure is that for NZETRatios greater than roughly 2.5, there is no significant reliability difference between the call ratios studied except for the $100 \%$ call ratio in the range $3.28 \leq$ NZETRatio $\leq 3.98$. In this range for very large call ratios, the NZET interacts with the market structure in such a way that unreliable periods are reduced and average reliability increases. Any non-contracted periods will upset this balance; the $80 \%$ call ratio and lower curves do not display this trend.

\subsection{RESULTS WITH SINUSOIDAL INPUT}

As with the square-wave command signal, sinusoidal command signal with asymmetric markets (Fig. 17) have the same predictable trends as those without SOC management (section 6.2) and the same features as the square-wave asymmetric structure. The predictable deviations are visible here as well (note that they are independent of energy content), along with the breakpoint associated with the command signal energy content. The main feature associated with the previous section is the increase in reliability after the breakpoint; reliability in this range reaches a maximum of $6.7 \%$ (100\% call ratio) to $11 \%$ (10\% call ratio) greater than section 6.2 (Fig. 18). Nearly all regions result in greater reliability than the results of 6.2. 


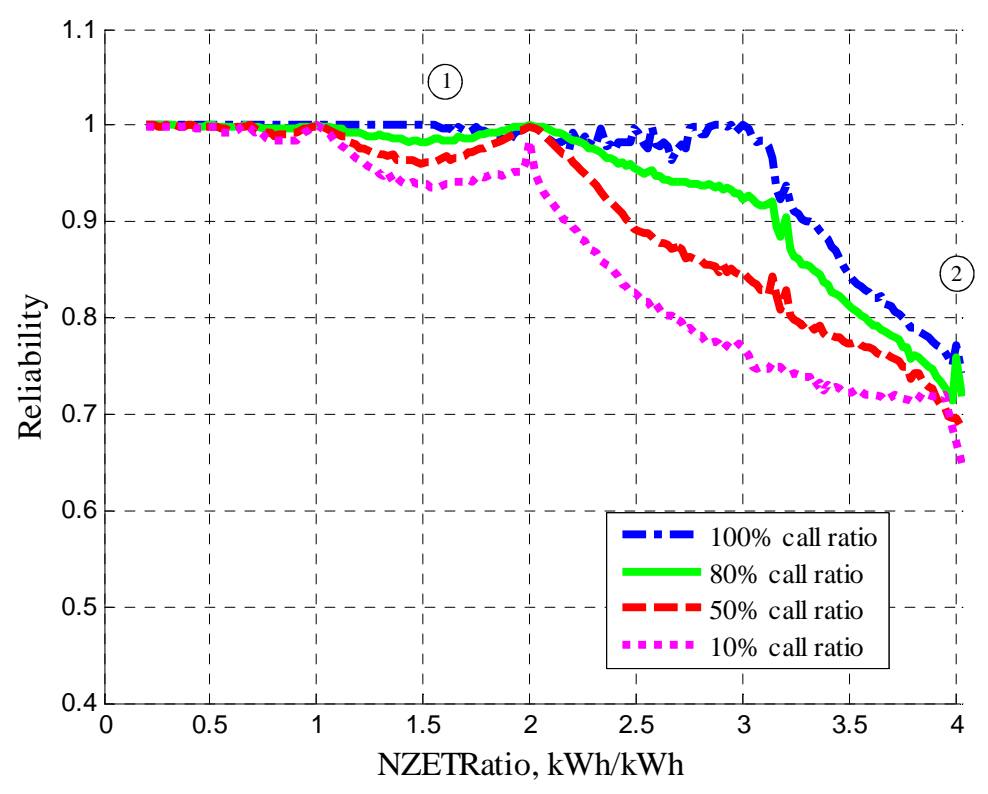

Fig. 17: Reliability vs. NZETRatio for three call ratio values and sinusoidal input with asymmetric market

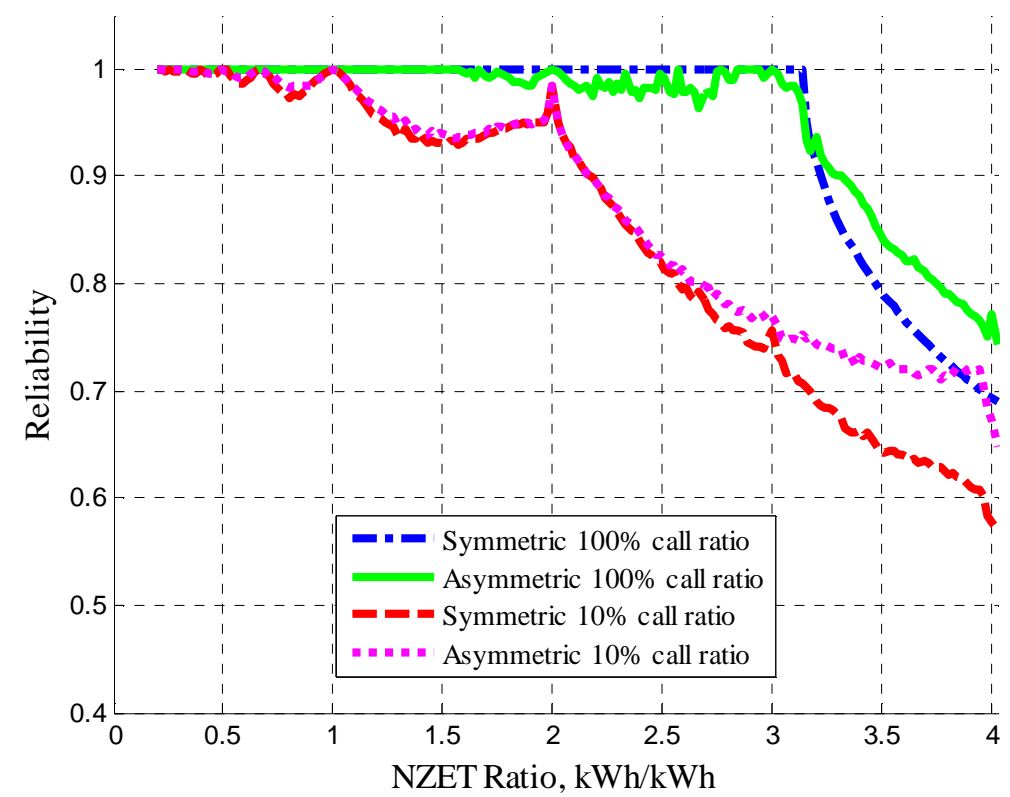

Fig. 18: Comparison of sinusoidal command signal results with asymmetric and simple symmetric markets

Call ratio is more important with a sinusoidal input than with square-wave. Although the reliability difference between curves in converging, it does not reach parity in the range studied. As before, higher call ratios command a higher reliability. Also, while there is a reliability 
difference among call ratios in this case, it should be noted that the least-reliable sinusoidal command signal provides greater reliability than the most-reliable square-wave signal over almost the entire range studied. Because of this, the sinusoidal command signal is the most advantageous for ES devices with this market structure. 


\subsection{DisCUSSION}

\subsection{Evaluation OF METHODS FOR IMPROVING ES DEVICE RELIABILITY}

\subsubsection{NZET REDUCTION}

The first solution to improve ES device reliability is to decrease the NZET. For the same ES device size, a decreased NZET would decrease NZETRatio by the same factor. NZETRatio $\leq 1$ or $\pi / 2$ (for square or sinusoidal command signal, respectively) would be the ideal choice, because devices with any call ratio would be very reliable. Practical implementation of NZET reduction is uncertain, however. In order to decrease NZET of an AGC signal, the energy content of the signal must be altered. Since this reduced-NZET signal will not correct for the entire energy content necessary to balance the grid, it is unclear how useful such a signal would be to the ISO/BA. Until it is apparent that this new signal can accommodate some significant portion of the full AGC signal energy, NZET reduction may not be the most viable method to increase ES device reliability.

\subsubsection{SOC MANAGEMENT}

The proposed SOC management strategy of non-contract charging provides an alternate method to increase ES device reliability. This is beneficial because reliability of ES can be increased relative to a simple symmetric structure while using the same command signal. To utilize this method, however, each ES device must be able to transfer between A/S markets and the energy market while still monitoring contracts and bids. While SOC management shows greater resource reliability than the simple symmetric structure, its implementation may counteract the potential benefits. By virtue of the device-level management, if a device discharges in an A/S contract, it will recharge the next time it is not contracted. If these contract 
periods are adjacent, the discharge to provide frequency regulation could be negated by the subsequent recharge.

\subsubsection{ASYMMETRIC REGULATION MARKETS}

The third and most valuable option is to have an asymmetric A/S market structure. This will allow for similar or better resource reliability than the symmetric structure (Fig. 19) without the drawbacks of device-level SOC management. An asymmetric structure would allow each device to manage its own SOC in a way that is beneficial to both the device and grid stability. Also, as mentioned above, the control points used in this study were not optimized for the device, which indicates potential reliability gains with further study.

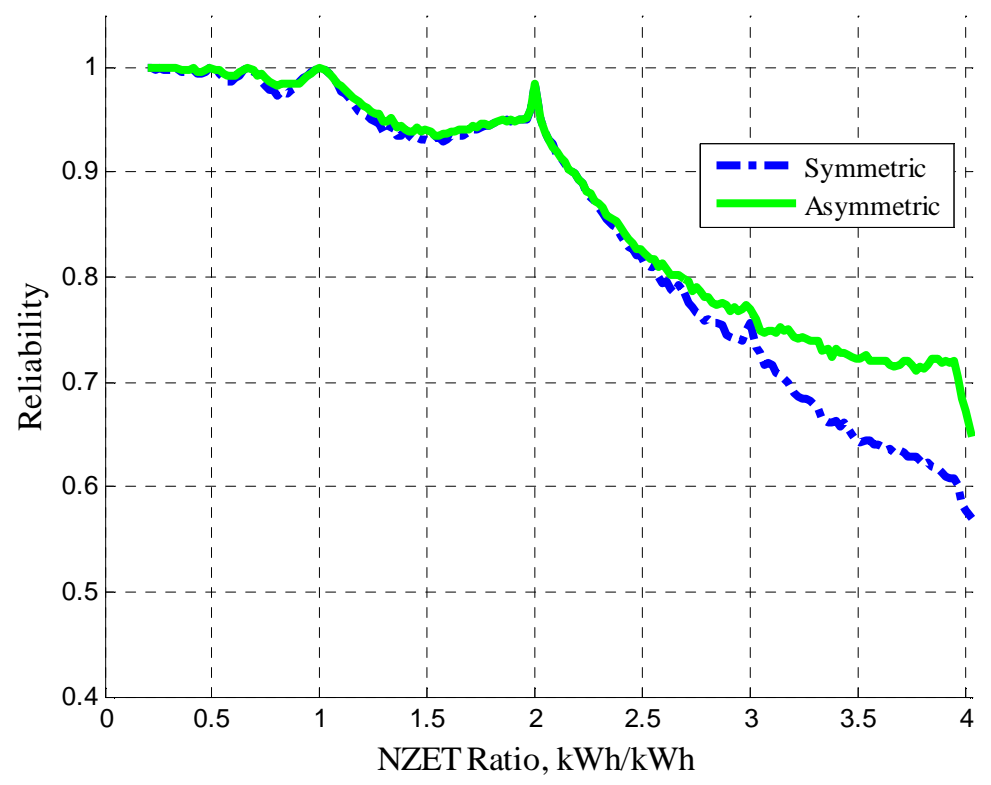

Fig. 19: Comparison of symmetric and asymmetric market structures with sinusoidal input and $10 \%$ call ratio 


\subsection{SyNTHESIZING DES SYSTEM RELIABILITY WITH FREQUENCY REGULATION}

Using the reliability of each type of signal, I will present two differing methods of aggregation (Fig. 2 and Fig. 3, shown below as Fig. 20 and Fig. 21, respectively) with the asymmetric structure. Fig. 20 is an example of the stacked call method, for which the squarewave command signal is an approximation. With this method, the power from the simulated AGC signal is divided into discrete sections, one for each individual ES device (in this example, five). Because the magnitude of power is equal for each ES device in the stack and reliability calculations are based on NZETRatio, the difference in reliability will depend only on the differing period. Based on the previous calculations, the AGC signal period in Fig. 20 equates to NZETRatio $=3$. Therefore, the contract labeled 1 has NZETRatio $=3,2$ has NZETRatio $=3-$ 3/5, 3 has NZETRatio $=3-6 / 5$, and so on. Since the overall DES system reliability is defined by the least-reliable ES device, and the 1 ES device has NZETRatio $=3$ (for a possible reliability of no greater than $75.0 \%$ (Fig. 14)), a DES system using the stacked call method in this case has an overall reliability of up to $75.0 \%$. 


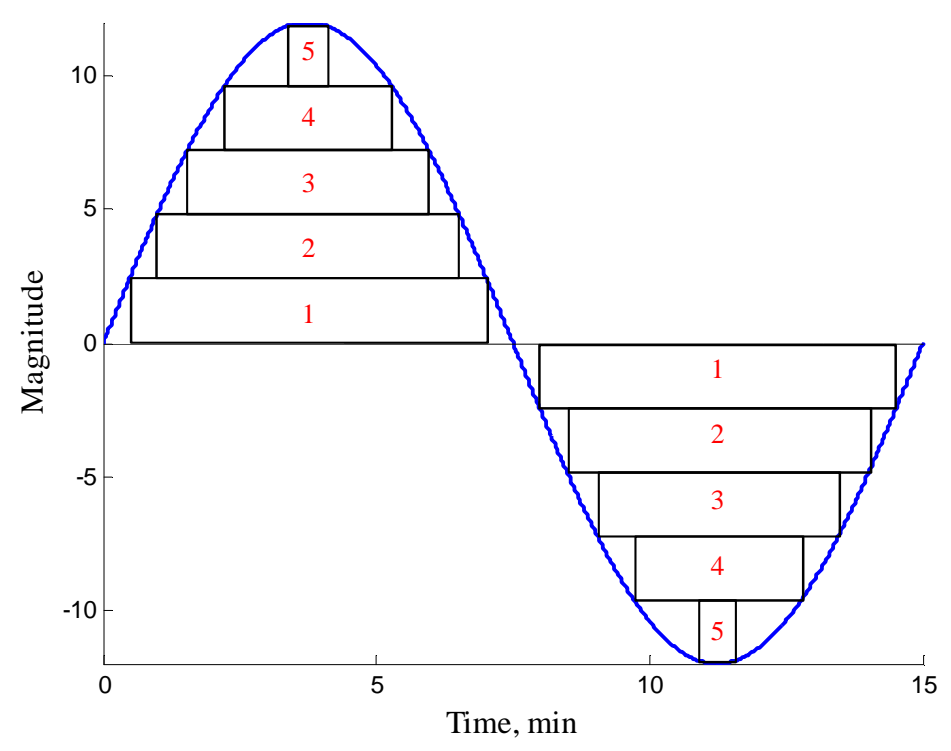

Fig. 20: Stacked call method for DES signal distribution

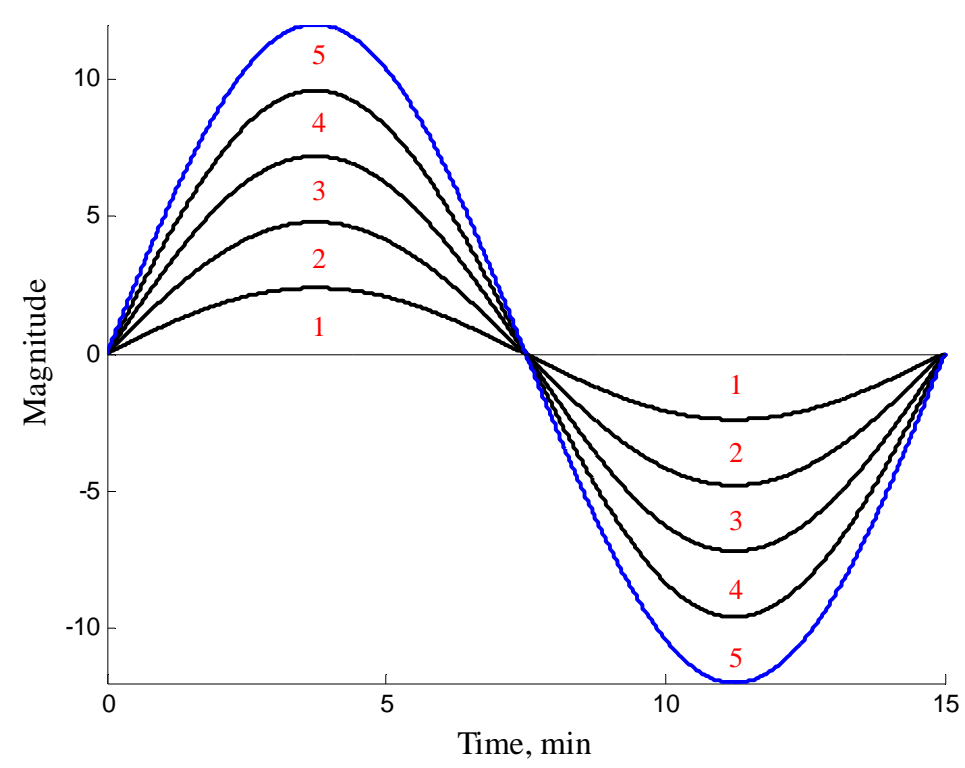

Fig. 21: Proportional call method for DES signal distribution

Fig. 21 is an illustration of the second utilization method, which was approximated with the sinusoidal command signal: proportional call. In this method, the input signal is divided into time-variant sections (in this example, five). Because each of the five ES devices receives equal magnitude and the period for each is the same, all batteries are operating at NZETRatio $=3$. 
Referring to Fig. 17 for a sinusoidal input, NZETRatio $=3$ corresponds to maximum possible DES system reliability of $100 \%$. Contrasted with the stacked call reliability of $75.0 \%$, the proportional call method is the more reliable means for division of an AGC signal to DES.

\subsection{Benchmarking Against CURREnTly-Available AGC Signals}

Using historical regulation data from PJM and CAISO, the definitions and results of this study can be used to rate production signals on their ability to support DES systems [36], [39], [40]. Table 2 shows the maximum absolute power and calculated NZET for PJM and CAISO standard regulation signals over a 24 -hour period on $7 / 23 / 11$ and $8 / 31 / 11$, respectively. The calculated NZET for the PJM signal is not an exact value because the signal energy did not return to zero within the period studied.

Table 2: Pertinent specifications for regulation signals over a 24-hour period

\begin{tabular}{|c|c|c|}
\hline & Max. Abs. Power, MW & Calculated NZET, hr \\
\hline PJM & 1475 & $>21.7$ \\
\hline CAISO & 238 & 8.25 \\
\hline
\end{tabular}

In order to have only DES systems contracted to perform regulation during that period, the regulation signal must be divided proportionally among enough systems to reach the maximum power. Assuming each system is equivalent to the world's largest commercial lithium-ion DES system (as of October 2011), with power capacity of 32MW and energy capacity of $8 \mathrm{MWh}$, the minimum number of contracted systems can be calculated to be 47 for the PJM standard regulation signal and 8 for the CAISO signal [13], [41]. Table 3 shows the NZETRatio for the DES system using the rated energy capacity. Table 4 shows the necessary 
capacity for each DES device to obtain a NZETRatio of 3, which is the largest realistic NZETRatio to guarantee high reliability for proportional call. This calculation uses the same number of contracted systems as Table 3 .

Table 3: NZETRatio for world's largest commercial DES system

\begin{tabular}{|c|c|}
\hline & NZETRatio for 8MWh DES \\
\hline PJM & 85 \\
\hline CAISO & 30.7 \\
\hline
\end{tabular}

Table 4: Necessary energy capacity to obtain NZETRatio $=3$

\begin{tabular}{|c|c|}
\hline & Necessary Energy Capacity, MWh \\
\hline PJM & 227 \\
\hline CAISO & 81.8 \\
\hline
\end{tabular}

The necessary energy capacity required for a DES system to achieve workable reliability with the standard regulation signal from either ISO is much larger than is presently feasible. Even for the current largest commercial lithium-ion DES system, the distributed NZETRatio is at minimum an order of magnitude above acceptable values. For DES systems to be viable in regulation markets, the ISO/BA will need command signals with much less energy content. Currently, PJM has a non-production Frequency Only (FO) command signal that is based on the AGC but without the tie error component [36]. This dramatically reduces both the required maximum power and the NZET. Table 5 shows results for necessary DES energy capacity to achieve NZETRatio = 3 with a 32MW DES system as well as calculated NZETRatio for a 32MW, 8MWh DES system using PJM's FO command signal for a 24-hour period on 8/31/11. These values are much more technically feasible, although the value of the FO signal is not yet defined. 
Table 5: Necessary energy capacity and NZETRatio for FO command signal

\begin{tabular}{|l|c|c|}
\hline & $\begin{array}{c}\text { Necessary Energy } \\
\text { Capacity, MWh }\end{array}$ & $\begin{array}{c}\text { NZETRatio for } \\
\text { 8MWh DES }\end{array}$ \\
\hline PJM FO & 5.25 & 1.97 \\
\hline
\end{tabular}

Several other studies have used similar energy-storage-specific regulation command signals. In an early work, Kempton et al. suggested that EDVs could rebalance the Area Control Error (ACE) directly, rather than through the AGC signal [22]. Hawkins used a specialized signal that had many more charge-discharge cycles than the traditional AGC, allowing smaller offsets from nominal SOC and was therefore better suited to small-capacity ES devices. The study also proposed official Automatic Storage Control and Automatic Load Control signals to better contract DES systems [30]. Quinn et al. simulated filtering and feedback control of the ACE to create a signal that increased reliability of DES systems over the traditional AGC signal. They concluded that this type of signal would be necessary to maintain system reliability [33]. Entriken et al. also proposed a frequency-specific signal by correcting the energy bias periodically. This lagging bias correction every five minutes greatly reduced the energy content of the signal [32]. In a subsequent study, a 30-second correction was added to the original correction as a secondary filter, further decreasing the energy content as well as decreasing the necessary power capacity [42]. Similarly, Brooks proposed a Processed ACE (PACE) signal for CAISO regulation, in which he filtered out the energy offset from the CAISO ACE. This produced a long-term average net-zero-energy signal, which was shown to be valuable to energylimited DES systems [24]. 


\subsection{AGGREGATION TO IMPROVE DES RELIABILITY}

Table 6 displays the calculated NZETRatio for a single automotive-type battery commanded by the PJM FO signal. Using proportional call and asymmetric market structure (Fig. 17), this NZETRatio is two orders of magnitude greater than the largest acceptable NZETRatio. It is also much larger than that of the studied DES system with standard regulation signals (Table 3). This NZETRatio would provide reliability far below any displayed in this study, which is why aggregation of ES devices is necessary for connection to the grid to be viable.

Table 6: NZETRatio for DES and individual ES device with FO command signal [33]

\begin{tabular}{|c|c|}
\hline & $\begin{array}{c}\text { NZETRatio for 10kWh } \\
\text { vehicle battery }\end{array}$ \\
\hline PJM FO & 1574 \\
\hline
\end{tabular}

Further aggregation, beyond the necessary minimum, can be used to decrease NZETRatio and therefore increase reliability. The NZETRatio for two aggregated 10kWh automotive-type batteries is 787 , a factor of two lower than a single battery. While this is still unacceptable, the trend continues with added devices. Due to (1), as the DES capacity increases, NZETRatio decreases proportionally. In association with proportional call and asymmetric markets, an increase in DES capacity is another method to increase system reliability. 


\subsection{Conclusions}

This study has developed a model of the behavior of distributed energy resources under a model of ancillary services contracting. Incorporated are analyses of the reliability of DES systems under various grid conditions, including call ratio and the energy structure of the AGC signal.

The model was used to determine relationships between the characteristics of the AGC signal that commands the individual energy storage resources and the resulting reliability of the system. The reliability of the DES can be characterized based on the NZETRatio of the AGC signal and the call ratio for both square-wave and sinusoidal power commands. Analysis of these power commands demonstrates that the sinusoidal power command, associated with aggregation via proportional call, provides the highest system reliability in nearly all ranges and market structures studied. Furthermore, an asymmetric A/S market structure is the most valuable method for increasing resource reliability, with NZET reduction and device-level SOC management as other potential approaches. The reliability of the DES systems in meeting the power commands is shown to decline with NZETRatios $>\sim 2$ as well as with call ratio.

Analysis of production AGC signals have shown that even the largest commercial lithium-ion DES system cannot adequately participate in commercial markets. This same system, however, provides enough energy storage to participate in the experimental PJM FO market. This market is characterized by a low-energy AGC signal, which effectively decreases the NZET from that of the standard signal.

Previous studies have assumed that automotive batteries are especially capable of regulation services because the net energy requirement eventually nets to zero or battery discharge will be minimal [6], [18], [22], [27]. This paper has shown that this is not necessarily 
true and has derived the guidelines for NZETRatio and call ratio that BAs can use to design command signals specific to DES systems. By implementing power commands that can enable DES systems to maintain high reliability, their value in improving the stability and value of the electric grid can be maximized. 


\subsection{REFERENCES}

[1] K. Parks et al., "Costs and Emissions Associated with Plug-In Hybrid Electric Vehicle Charging in the Xcel Energy Colorado Service Territory," Nat. Renewable Energy Lab., Golden, CO, Tech. Rep. NREL/TP-640-41410, 2007.

[2] W. Kempton and S. E. Letendre, "Electric Vehicles as a New Power Source for Electric Utilities," Transportation Research Part D, vol. 2, no. 3, pp. 157-175, 1997.

[3] J. Neubauer and A. Pesaran, "NREL's PHEV/EV Li-ion Battery Secondary-Use Project," presented at the Advanced Automotive Batteries Conf., Orlando, FL, 2010.

[4] J. Neubauer et al., "Secondary Use of PHEV and EV Batteries - Opportunities \& Challenges," presented at the Advanced Automotive Battery Conf., Orlando, FL, 2010.

[5] E. Wood et al., "Investigation of battery end-of-life conditions for plug-in hybrid electric vehicles," J. Power Sources, vol. 196, pp. 5147-5154, Feb. 2011. doi: 10.1016/j.jpowsour.2011.02.025

[6] W. Kempton et al., "A Test of Vehicle-to-Grid (V2G) for Energy Storage and Frequency Regulation in the PJM System," Univ. Delaware, Pepco Holdings, PJM Interconnect, Green Mountain College, 2008.

[7] S. Kamboj et al., "Deploying Power Grid-Integrated Electric Vehicles as a Multi-Agent System," in Proc. of 10th Int. Conf. on Autonomous Agents and Multi-agent Systems Innovative Applications Track (AAMAS 2011) 2011 (C) International Foundation for Autonomous Agents and Multiagent Systems.

[8] S. D. Jenkins et al., "Utilization and effect of plug-in hybrid electric vehicles in the United States power grid," in IEEE Vehicle Power and Propulsion Conf., 2008 @ IEEE. doi: 10.1109/VPPC.2008.4677501.

[9] S. McElligot. (2010, November) Flywheels Set to Help Regulate Frequency on the U.S. Grid. TechSurveillance [Online]. Available: http://www.beaconpower.com

[10] K. Madani and J. R. Lund, "Modeling California's high-elevation hydropower systems in energy units," Water Resources Research, vol. 45, pp. 1-12, Sep. 2009. doi: 10.1029/2008WR007206

[11] E. Cready et al., "Technical and Economic Feasibility of Applying Used EV Batteries in Stationary Applications," Sandia Nat. Lab., Albuquerque, NM, Rep. SAND2002-4084, 2003.

[12] W. Kempton and J. Tomic, "Vehicle-to-grid power implementation : From stabilizing the grid to supporting large-scale renewable energy," J. Power Sources, vol. 144, pp. 280294, 2005. doi: 10.1016/j.jpowsour.2004.12.022 
[13] U. Wang. (2011, October 27) The World's Largest Lithium-Ion Battery Farm Comes Online [Online]. Available: http://www.forbes.com

[14] W. Kempton and T. Kubo, "Electric-drive vehicles for peak power in Japan," Energy Policy, vol. 28, no. 1, pp. 9-18, 2000.

[15] J. M. Eyer et al., "Energy Storage Benefits and Market Analysis Handbook," Sandia Nat. Lab., Albuquerque, NM, Rep. SAND2004-6177, 2004.

[16] F. Moura, "Driving energy system transformation with 'vehicle-to-grid' power," Int. Institute for Applied Systems Analysis, Laxenburg, Austria, Rep. IR-06-025, 2006.

[17] F. R. Kalhammer et al., "Status and Prospects for Zero Emissions Vehicle Technology," California Air Resources Board, Sacramento, CA, 2007.

[18] W. Kempton and J. Tomic, "Vehicle-to-grid power fundamentals: Calculating capacity and net revenue," J. Power Sources, vol. 144, no. 1, pp. 268-279, Jun. 2005. doi: 10.1016/j.jpowsour.2004.12.025

[19] J. Tomic and W. Kempton, "Using fleets of electric-drive vehicles for grid support," $J$. Power Sources, vol. 168, no. 2, pp. 459-468, Jun. 2007. doi:

10.1016/j.jpowsour.2007.03.010

[20] B. Kramer et al., "A Review of Plug-in Vehicles and Vehicle-to-Grid Capability," in Industrial Electronics, Orlando, FL, 2008, pp. 2278 - 2283. doi: 10.1109/IECON.2008.4758312

[21] P. Denholm and W. Short, "An Evaluation of Utility System Impacts and Benefits of Optimally Dispatched Plug-In Hybrid Electric Vehicles," Nat. Renewable Energy Lab., Golden, CO, Tech. Rep. NREL/TP-620-40293, 2006.

[22] W. Kempton et al., "Vehicle-to-Grid Power: Battery, Hybrid, and Fuel Cell Vehicles as Resources for Distributed Electric Power in California," Inst. of Transportation Studies, Univ. California, Davis, CA, Rep. UCD-ITS-RR-01-03, 2001.

[23] C. Quinn et al., "The effect of communication architecture on the availability, reliability, and economics of plug-in hybrid electric vehicle-to-grid ancillary services," J. Power Sources, vol. 195, no. 5, pp. 1500-1509, Mar. 2010. doi: 10.1016/j.jpowsour.2009.08.075

[24] A. N. Brooks, "Vehicle-to-Grid Demonstration Project: Grid Regulation Ancillary Service with a Battery Electric Vehicle," AC Propulsion, Inc., San Dimas, CA, Contract 03-313, Final Rep., Dec. 2002.

[25] S. Kamboj et al., "Exploring the formation of Electric Vehicle Coalitions for Vehicle-ToGrid Power Regulation," AAMAS Workshop on Agent Technologies for Energy Systems, 2010. 
[26] K. Clement-Nyns et al., "The impact of vehicle-to-grid on the distribution grid," Electric Power Systems Research, vol. 81, no. 1, pp. 185-192, 2011. doi: 10.1016/j.epsr.2010.08.007

[27] A. Brooks and T. Gage, "Integration of Electric Drive Vehicles with the Electric Power Grid -- a New Value Stream," in EVS-18, Berlin, 2001.

[28] T. B. Gage, "Development and Evaluation of a Plug-in HEV with Vehicle-to-Grid Power Flow," AC Propulsion, Inc., San Dimas, CA, Rep. ICAT 01-2, 2003.

[29] M. D. Galus et al., "On integration of plug-in hybrid electric vehicles into existing power system structures," Energy Policy, vol. 38, no. 11, pp. 6736-6745, Nov. 2010. doi: 10.1016/j.enpol.2010.06.043

[30] D. Hawkins, "Integration of Energy Storage Technology White Paper: Identification of Issues and Proposed Solutions," CAISO, 2008.

[31] R. Sioshansi and P. Denholm, "The Value of Plug-In Hybrid Electric Vehicles as Grid Resources," Energy Journal, vol. 31, no. 3, pp. 1-23, 2010.

[32] R. Entriken et al., "Prototype Method for Analyzing Regulation by Limited Energy Storage,” EPRI, Palo Alto, CA, 2010.

[33] C. Quinn et al., "An Evaluation of State-of-Charge Limitations and Actuation Signal Energy Content on Plug-in Hybrid Electric Vehicle, Vehicle-to-Grid Reliability, and Economics systems," IEEE Trans. on Smart Grid, vol. 3, pp. 483-491, Mar. 2012. doi: 10.1109/TSG.2011.2168429

[34] C. Loutan and D. Hawkins, "Integration of Renewable Resources," CAISO, 2007.

[35] "Storage Participation in ERCOT," Texas Energy Storage Alliance, 2010.

[36] PJM Market-Based Regulation [Online]. Available: http://pjm.com/markets-andoperations/ancillary-services/mkt-based-regulation.aspx

[37] T. Gómez et al., "Ancillary Services Markets in California,” Univ. California, Berkeley, CA, Pub. LBNL-43986, 1999.

[38] Generation Availability Report [Online]. Available: http://www.nerc.com

[39] CAISO OASIS Database [Online]. Available: http://oasis.caiso.com

[40] T. Boston, “The Silver Bullet...Storage,” PJM Interconnection, 2011.

[41] A123 Systems Grid Solutions [Online]. Available: http://www.a123systems.com/08852ac7-4a96-47e8-b3d4-6696b89beefc/download.htm 
[42] N. Taheri and R. Entriken, "Prototype Method for Analyzing Regulation by Limited Energy Storage," EPRI, Palo Alto, CA, 2009.

Acknowledgement: "This material is based on work supported by the Department of Energy under Award Number 'DE-EE0002627'"

Disclaimer: "This report was prepared as an account of work sponsored by an agency of the United States Government. Neither the United States Government nor any agency thereof, nor any of their employees, makes any warranty, express or implied, or assumes any legal liability or responsibility for the accuracy, completeness or usefulness of any information, apparatus, product, or process disclosed, or represents that its use would not infringe privately owned rights. Reference herein to any specific commercial product, process, or service by trade name, trademark, manufacturer, or otherwise does not necessarily constitute or imply its endorsement, recommendation, or favoring by the United States Government or any agency thereof. The views and opinions of authors expressed herein do not necessarily state or reflect those of the United States Government or any agency thereof." 


\subsection{APPENDIX}

\subsection{CONTROL POINT ANALYSIS}

Fig. 22 shows the results of the analysis completed to determine the control points for use in the asymmetric structure study. As can be seen, reliability is lowest for the $50 \%-50 \%$ control points (where if the SOC is below 50\%, the ES device will charge only, and if it is above 50\%, the ES device will discharge only) and increases with broadening control point range up to $100 \%-0 \%$ control with the aforementioned $0.5 \%$ buffer. This control structure gives the best overall resource reliability. 

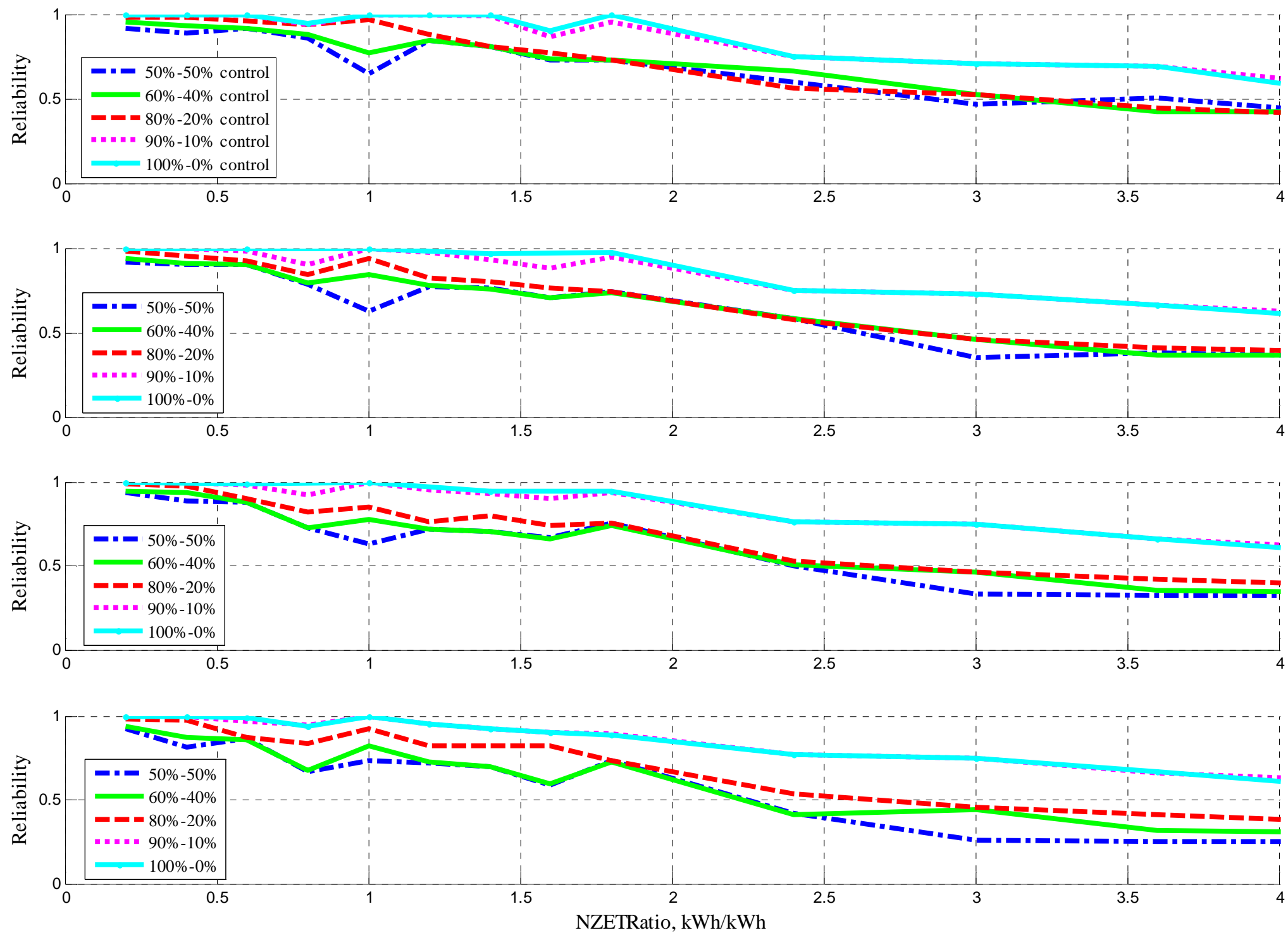

Fig. 22: Control point analysis for asymmetric structure study. From top to bottom: $100 \%$ call ratio, $80 \%$ call ratio, $50 \%$ call ratio, and $10 \%$ call ratio 


\subsection{EXPLANATION OF DEVIATIONS}

There are several deviations in this simulation that are due to the mathematic precision of computer software and will not be a factor in real-world applications. Certain discrete points in the simulations resulted in severe drops in reliability, while other points resulted in swift increases. This is caused by the relationship between NZET and contract length. Whenever the length of a contract is a multiple of the NZET, the SOC trace of each subsequent contract will be similar or identical to the first for each device, which could either artificially drop or raise the reliability at that point. This irregularity is not applicable to real-world applications because such a strict NZET would not occur; a real-world command signal is constantly varying in all aspects, so the probability of the NZET being an exact multiple of the contract length for several consecutive contracts is extremely low.

An important exception is the stark reliability increase at and around NZETRatio $=2$. This increase and decrease encompasses many NZETRatios and is indicative of real-world reliability values. The predictable reliability deviations for $0<$ NZETRatio $\leq 2$ (Fig. 8) can be explained with Fig. 23. This plot is based on the nominal energy absorbed or depleted at the end of one contracted period. The depth and shape of the valleys are dependent on call ratio and $t_{\text {deplete }}$ (which varies both with NZET and command signal structure), so these parameters cannot be matched analytically with the simulation results, but the position of the reliability peaks match this solution exactly. 


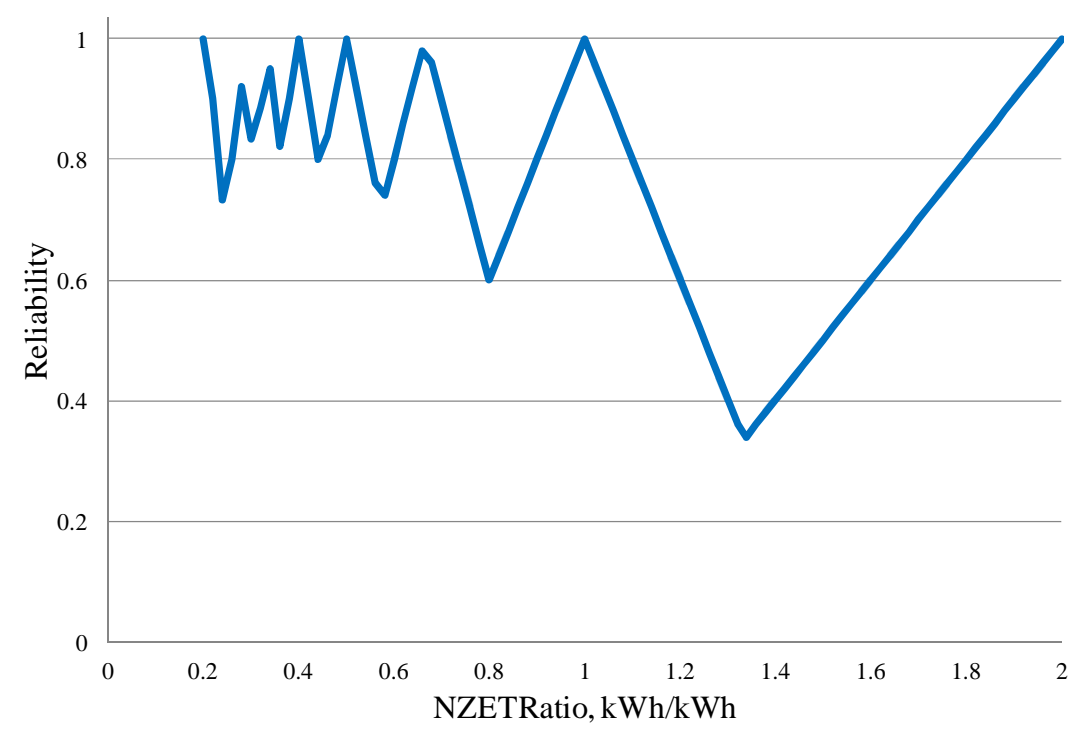

Fig. 23: Analytical explanation of the predictable reliability deviations

The reliability of DES for $\lim _{N Z E T R a t i o \rightarrow 2}$ is especially interesting because the reliability difference between maximum and minimum at this limit decreases dramatically for both squarewave and sinusoidal command signals. This NZETRatio value is specific to this model, however. NZETRatio $=2$ for this study is equivalent to a NZET $=10$ minutes, which is equivalent to the contract period. As mentioned above, it is not practical to assume real-world applications will follow each point of the simulation curves exactly, but the numerous points trending toward considerably higher reliability for NZET equal to contract period suggests that if NZET for DES can be controlled closely, it may be advantageous to have a setpoint near or equal to the length of the contract period. 